\title{
Integrated Quantum Photonics with Silicon Carbide: Challenges and Prospects
}

\author{
Daniil M. Lukin, Melissa A. Guidry, and Jelena Vučković@** \\ E. L. Ginzton Laboratory, Stanford University, Stanford, California 94305, USA
}

(Received 17 August 2020; accepted 2 November 2020; published 15 December 2020)

\begin{abstract}
Optically addressable solid-state spin defects are promising candidates for storing and manipulating quantum information using their long coherence ground-state manifold; individual defects can be entangled using photon-photon interactions, offering a path toward large-scale quantum photonic networks. Quantum computing protocols place strict limits on the acceptable photon losses in the system. These lowloss requirements cannot be achieved without photonic engineering, but are attainable if combined with state-of-the-art nanophotonic technologies. However, most materials that host spin defects are challenging to process: as a result, the performance of quantum photonic devices is orders of magnitude behind that of their classical counterparts. Silicon carbide $(\mathrm{SiC})$ is well suited to bridge the classical-quantum photonics gap, since it hosts promising optically addressable spin defects and can be processed into SiC-on-insulator for scalable, integrated photonics. In this paper, we discuss recent progress toward the development of scalable quantum photonic technologies based on solid-state spins in silicon carbide, and discuss current challenges and future directions.
\end{abstract}

DOI: 10.1103/PRXQuantum.1.020102

\section{INTRODUCTION}

Quantum information processing (QIP) is among the most rapidly developing areas of science and technology. It is perhaps the final frontier in the quest to harness the fundamental properties of matter for computation, communication, data processing, and molecular simulation. Any physical system governed by the laws of quantum mechanics can in principle be a candidate for QIP; to date, however, the most advanced QIP demonstrations have been implemented via superconducting qubits [1], trapped ions and atoms $[2,3]$, and photons (via linear-optical quantum computing) [4]. Recently, optically addressable crystal defects have emerged as a novel platform for QIP [58], interfacing some of nature's best quantum memories (a protected solid-state spin [9-11]) with a robust flying qubit (photon) that can transport the quantum information [12]. Notably, solid-state defects lend themselves to on-chip integration, promising future scalability. Optically addressable spin defects are thus noteworthy candidates for several QIP proposals, including network-based quantum computing [13-15], cluster state generation [16-18], and quantum communications $[19,20]$.

\section{*jela@stanford.edu}

Published by the American Physical Society under the terms of the Creative Commons Attribution 4.0 International license. Further distribution of this work must maintain attribution to the author(s) and the published article's title, journal citation, and DOI.
In recent years, the field of defect-based QIP has made extraordinary strides toward realizing such proposals. Breakthroughs include the demonstration of longdistance entanglement of solid-state spins [12]; highfidelity single-shot readout $(F>0.9995)$ of a color-center spin state and memory-enhanced quantum communication [21]; nanophotonic quantum memories based on rare-earth ensembles [22]; entanglement distillation between distant electron-nuclear two-qubit nodes [23]; and a ten-qubit quantum register based on nuclear spins coupled to a single color center, with single-qubit coherence exceeding one minute [10]. Although scalability is cited as a strength of optically addressable spin defects, the field has yet to demonstrate a breakthrough toward this end. So far, entanglement has been realized between at most two optically connected color centers, whether in fiber networks [12] or on a chip [24]. A central issue is the efficient interaction between defects and photons. The photon emission of a dipole source is difficult to direct into a single optical mode, a prerequisite for photon interference. The resulting low collection efficiencies translate into prohibitively low rates of higher-dimensional entanglement generation. However, by integrating a defect with a nanophotonic cavity, one can greatly enhance the photon emission rate into the cavity mode via the Purcell effect, thereby funneling the majority of emitted photonics into the desired optical channel. This powerful technique has, for instance, enabled single-shot readout of single rare-earth ions [25,26], which are too dim outside of a cavity to even observe individually. Many of the aforementioned recent breakthroughs in 
defect-based QIP have been enabled by integration with nanophotonics $[21,22,24]$.

Defect-based integrated quantum photonics $[7,8]$ is thus a recent and exciting union of two distinct and rapidly developing fields: the study of quantum spintronics [5,6] and the development of classical integrated photonics [2730]. In this paper, we discuss the challenges and prospects for the field in context of the recent advances in silicon carbide $(\mathrm{SiC})$. Silicon carbide hosts a wide range of optically active defects [31] and is amenable to CMOS-compatible photonics fabrication technologies [32-34], and is thus a key candidate in implementing fully integrated quantum photonic circuits. We begin with a brief summary of the fundamental requirements and challenges of defect-based QIP, followed by a summary of the state of the art in SiC defects and in SiC photonics. We then discuss the feasibility of overcoming key challenges to develop scalable integrated quantum photonic circuits.

\section{MOTIVATION FOR COMBINING SPIN DEFECTS WITH NANOPHOTONICS}

An optically addressable defect, illustrated in Fig. 1(a), features a ground-state manifold with a long coherence time that can emit spin-entangled photons. This manifests as spin-dependent photon emission, where either the polarization or frequency of the photon encodes the electron spin state (alternatively, time-bin entanglement can be used [36]). The electronic spin can also be coupled to one or more nearby nuclear spins $[6,10,37]$. Thus, the defect can serve as a multiqubit register, for applications in error-corrected quantum computation [as part of a quantum photonic network, Fig. 1(b)] [15] or as a source of photonic cluster states $[16,17]$ for quantum communications and linear-optical quantum computing.

Spin-entangled optical photons are ideal carriers of quantum information for generating remote entanglement. Their high energy renders them insensitive to decoherence at room temperature (enabling routing of quantum information via the same commercial fibers that route classical data) and makes it possible to measure them with high quantum efficiency [38]. However, optical photons are difficult to confine within an integrated circuit. In contrast with microwave photons whose efficient confinement in metal wires enables photolithographically defined superconducting qubit circuits, optical photons are only weakly confined in dielectric structures using refractive index contrast. Distributed Bragg reflectors, such as photonic crystals, can be used to engineer a fully reflecting boundary to confine and route light [39], but the high photon energy dictates the small feature size of these confining structures, requiring more sophisticated nanofabrication methods. Furthermore, since three-dimensional photonic crystals [39] (which create a complete $4 \pi$ steradian bandgap) are not yet practical, all means to confine light on a chip still rely on weak confinement via total internal reflection along at least one spatial dimension. Although a waveguide based on total internal reflection is (a)

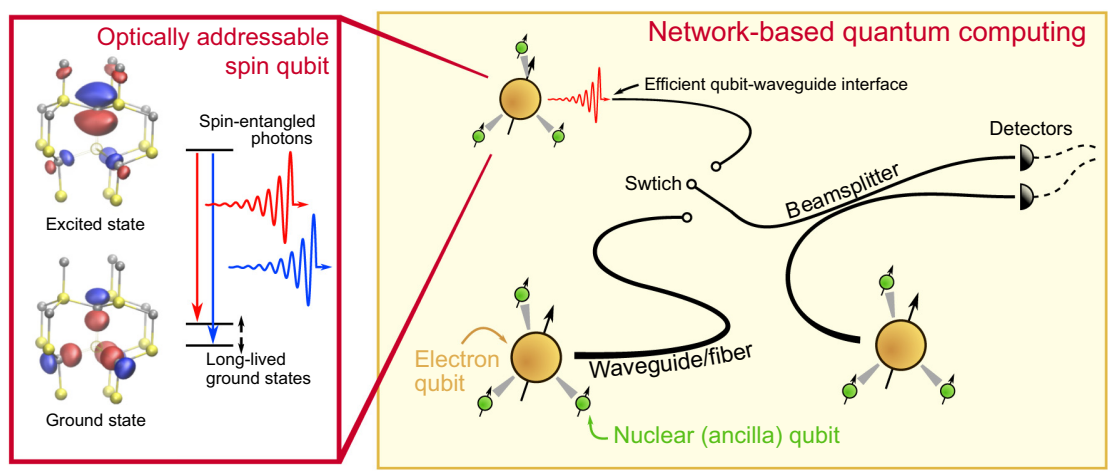

(c)

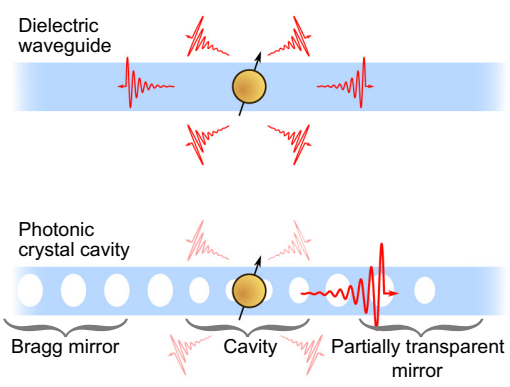

FIG. 1. Quantum photonics with optically addressable spins. (a) A suitable defect features a spin-selective optical transition, where a photon degree of freedom (i.e., polarization, frequency, or time bin) is entangled with a ground-state spin featuring a long coherence time. The defect's optical lifetime is determined by the magnitude of its optical dipole moment, which in turn is dictated by the orbital structure of the excited and ground states. (b) A quantum photonic network consists of multiqubit registers, each consisting of an optically addressable electron spin strongly coupled to nearby nuclear spins. The registers are integrated together in a network via an efficient waveguide or fiber interface. The network is equipped with beamsplitters and switches (which may be one and the same depending on the implementation) for long-distance entanglement and circuit reconfigurability. Low losses at all stages (including efficient photon collection from the defect, low-loss photon propagation in the waveguide, and high detector efficiency) are essential for fault-tolerant computation, efficient quantum simulation, and long-distance quantum communications. (c) Because of the weak confinement of optical photons in dielectric structures, light from a quantum emitter does not couple efficiently to a simple dielectric waveguide. Instead, a nanophotonic cavity or a slow-light waveguide mode must be used to enhance the emission into the waveguide mode via the Purcell effect. Orbital graphic in (a) adapted from Ref. [35]. 
a theoretically lossless and experimentally practical structure for routing photons on chip, it is not straightforward to make a defect efficiently emit photons into the waveguide in the first place. This can be intuitively understood by decomposing the dipole radiation pattern in the planewave basis and noting that only a modest fraction of dipole emission goes into those plane-wave modes that get totally internally reflected, while the rest are scattered into a free-space continuum of modes. In order to increase the fraction of photons emitted into the desired mode (referred to as the $\beta$ factor), one must rely on more advanced techniques, through careful control over the electromagnetic local density of states (LDOS). By embedding an emitter into a nanophotonic cavity that is coupled strongly to a waveguide, or by enhancing the LDOS in the waveguide itself [40], it is possible to enhance the defect's single-mode emission while maximally suppressing all other scattering [Fig. 1(c)]. Using this approach, $\beta$ factors approaching unity (to the point where they are negligible compared to other losses) have been achieved $[21,25]$. Since photon emission is reciprocal to photon absorption, a defect well coupled to a waveguide is equivalently suitable for reflection-based spin-photon entanglement via dipole-induced transparency [41].

The control of the LDOS of the quantum emitter not only minimizes the undesirable interactions with freespace modes, but also reduces the effects of other emitter nonidealities caused by its interaction with the solidstate environment. There are several mechanisms that degrade a defect's performance as a spin-photon interface. First, environmental fluctuations induce decoherence of the emitted photons, manifesting as the broadening of optical linewidths beyond the Fourier-transform limit (thus reducing the emitted photon indistinguishability). Second, many emitters have nonradiative pathways, via for example phonon-assisted spin-mixing transitions [42]. As a result, most emitters have a nonunity quantum efficiency, meaning not every excitation yields a photon. Third, coupling of the optical transition to optical phonons creates an additional decay pathway, whereby a photon is emitted in combination with one or more phonons. This emission is broad in spectrum (greater than $0.1 \mathrm{eV}$ ) and cannot be used for entanglement purposes. The fraction of "useful"- direct - emission into the zero-phonon line (ZPL) is referred to as the Debye-Waller factor (DWF). The DWF depends on the electronic orbital structure of the defect, and thus varies greatly for different defect types. Modification of the LDOS via, for example, integration of the defect into a cavity, enhances the emission rate into the ZPL via the Purcell effect, thus boosting the effective DWF and the quantum efficiency of the defect. Furthermore, since the emission enhancement is accompanied by lifetime reduction, the negative effect of spectral diffusion and homogeneous broadening on photon indistinguishability is also reduced.
The key purpose of combining a spin defect with photonic resonators is thus to increase its interaction with indistinguishable photons to enable efficient entanglement of remote defects. Here, "remote" signifies a distance greater than approximately $10 \mathrm{~nm}$ [43], beyond which direct dipole-dipole interaction between two defects is too weak.

\section{STATE OF THE ART IN SiC SPINTRONICS AND PHOTONICS}

\section{A. Optically addressable spins in $\mathrm{SiC}$}

Silicon carbide, in its numerous polytypes, has proven to be a versatile host to optically addressable, long-lived spin qubits $[5,6,31]$. Here, we briefly review the developments in spin-based quantum technologies in $\mathrm{SiC}$. We focus on the two most well-studied color centers to date, the silicon vacancy and the divacancy; we also highlight several emergent defects, such as the chromium ion, that may offer new functionalities. A summary of the properties of select defects in $\mathrm{SiC}$ is presented in Table I. We note here that the more complex $\mathrm{SiC}$ polytypes like $4 \mathrm{H}$ and $6 \mathrm{H}$ have multiple inequivalent lattice sites within a crystal unit cell [illustrated in Fig. 2(a)]. Consequently, each defect in Table I is a family of several defect types with similar but not identical properties. In addition to inequivalent lattice sites, rotational symmetries of the crystal give rise to multiple orientations of the same defect, the properties of which are otherwise identical.

\section{The silicon vacancy}

The negatively charged silicon vacancy $\left(\mathrm{V}_{\mathrm{Si}}^{-}\right.$, written $\mathrm{V}_{\mathrm{Si}}$ henceforth), a single missing silicon atom with an extra electron at the vacancy site, has been observed in the $4 \mathrm{H}$, $6 \mathrm{H}$, and $15 \mathrm{R}$ polytypes of $\mathrm{SiC}$. Its electronic configuration is modeled by five active electrons (three holes) resulting in a unique spin- $\frac{3}{2}$ system [75], which has enabled novel sensing protocols $[76,77]$ and the realization of a spin qudit [78]. In single isolated defects, a spin-coherence time of $0.8 \mathrm{~ms}$ has been measured at $4 \mathrm{~K}$ [35]. In $\mathrm{V}_{\mathrm{Si}}$ ensembles, a spin-coherence time as high as $T_{2}=20 \mathrm{~ms}$ has been observed using dynamic decoupling techniques [47]. Numerous approaches to generate $\mathrm{V}_{\mathrm{Si}}$ have been studied, including irradiation using electrons [57,79], neutrons [80], and protons [73]. In an effort to optimize $\mathrm{V}_{\mathrm{Si}}$ generation, the impact of different irradiation approaches on spin coherence has been systematically investigated [81]. Direct laser writing [74], ion implantation [82], and proton beam writing [73] have been investigated for deterministic defect placement [Figs. 2(b) and 2(c)]. Recently, the theoretically predicted [83] excited state fine structure of the $\mathrm{V}_{\mathrm{Si}}$ was experimentally confirmed in both inequivalent lattice sites in $4 \mathrm{H}-\mathrm{SiC}[35,48]$, enabling high-fidelity spin initialization [35] via the spin-selective intersystem crossing 
TABLE I. Optically addressable spin defects in SiC.

\begin{tabular}{lcccccccc}
\hline \hline Defect & $\begin{array}{c}\mathrm{ZPL} \\
(\mathrm{nm})\end{array}$ & Polytype & DWF & $\begin{array}{c}\text { Inverse } \\
\text { lifetime } \\
(\mathrm{MHz})\end{array}$ & $\begin{array}{c}\text { Measured } \\
\text { linewidth } \\
(\mathrm{MHz})\end{array}$ & $\begin{array}{c}\text { Stark } \\
\text { shift } \\
(\mathrm{GHz})\end{array}$ & $\begin{array}{c}\text { Spin } T_{2} \\
(\mathrm{~ms})\end{array}$ & Refs. \\
\hline $\mathrm{V}_{\mathrm{Si}}^{-}$ & $862-917$ & $4 \mathrm{H}, 6 \mathrm{H}, 15 \mathrm{R}$ & $0.06-0.09$ & 27 & 51 & 200 & 20 & {$[35,44-54]$} \\
$\mathrm{V}_{\mathrm{Si}} \mathrm{V}_{\mathrm{C}}^{0}$ & $1078-1132$ & $4 \mathrm{H}, 6 \mathrm{H}, 3 \mathrm{C}$ & 0.07 & 11 & 20 & 850 & 64 & {$[11,49,55-63]$} \\
$\mathrm{N}_{\mathrm{C}} \mathrm{V}_{\mathrm{Si}}^{-}$ & $1180-1468$ & $4 \mathrm{H}, 6 \mathrm{H}, 3 \mathrm{C}$ & $\ldots$ & 75 & $\ldots$ & $\cdots$ & $0.001\left(T_{2}^{*}\right)$ & {$[64-68]$} \\
$\mathrm{Cr}^{4+}$ & 1042,1070 & $4 \mathrm{H}$ & 0.75 & 0.002 & 31 & $\cdots$ & 0.081 & {$[69,70]$} \\
$\mathrm{V}^{4+}$ & $1278-1388$ & $4 \mathrm{H}, 6 \mathrm{H}$ & $<0.50$ & $0.9-14$ & 750 & $\cdots$ & $\cdots$ & {$[71,72]$} \\
\hline \hline
\end{tabular}

pathways $[42,83]$. The observation of narrow optical transitions [35,48,52], as shown in Fig. 2(d), allowed for the demonstration of highly indistinguishable photon emission with above-resonant driving [52] [Fig. 2(e)], an important step toward implementing cluster-state generation proposals $[17,84]$. The theoretically predicted first-order dc Stark shift [85] has been observed in ensembles [53] as well as in single defects [54]. The demonstrated tuning range

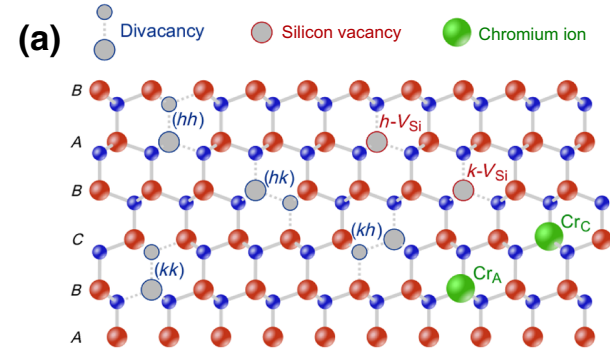

(d)

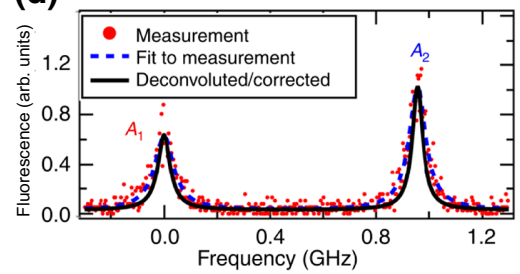

(g)

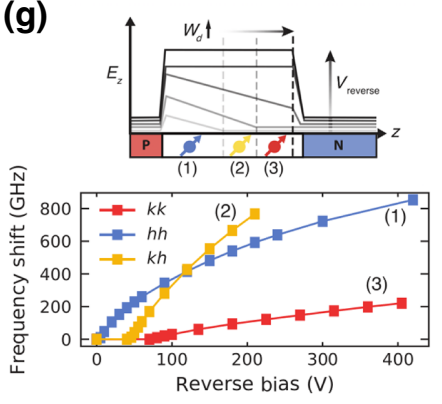

(e)

(h) (b)
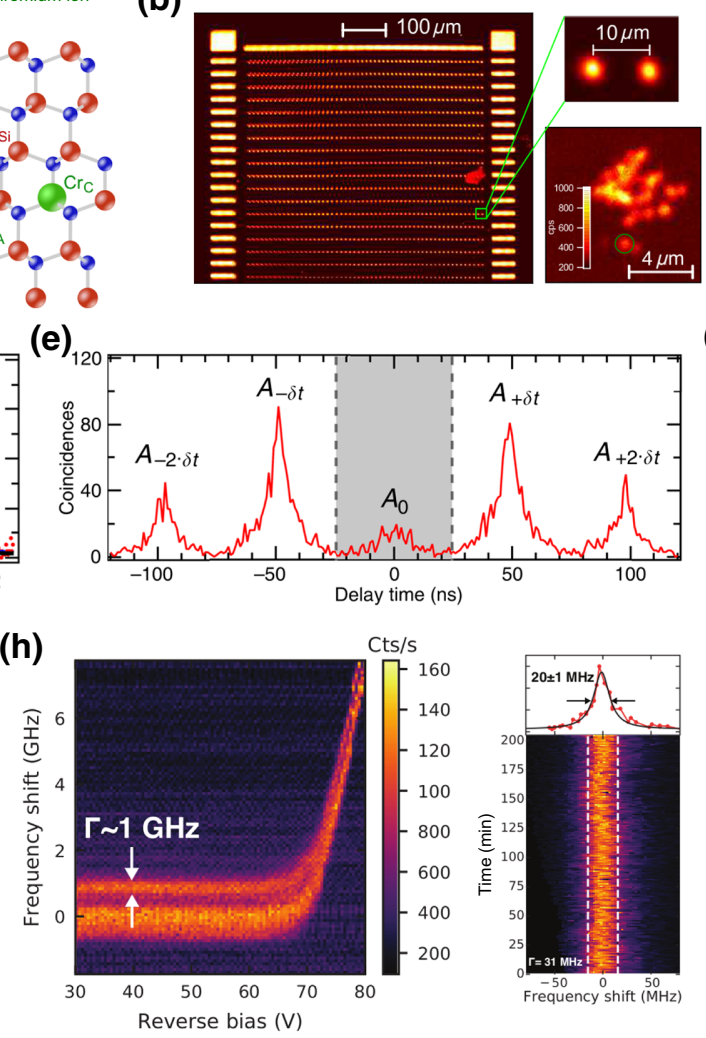

(c)

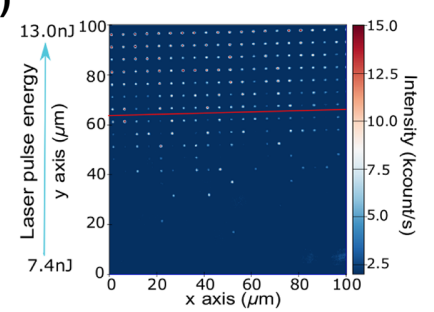

(f)
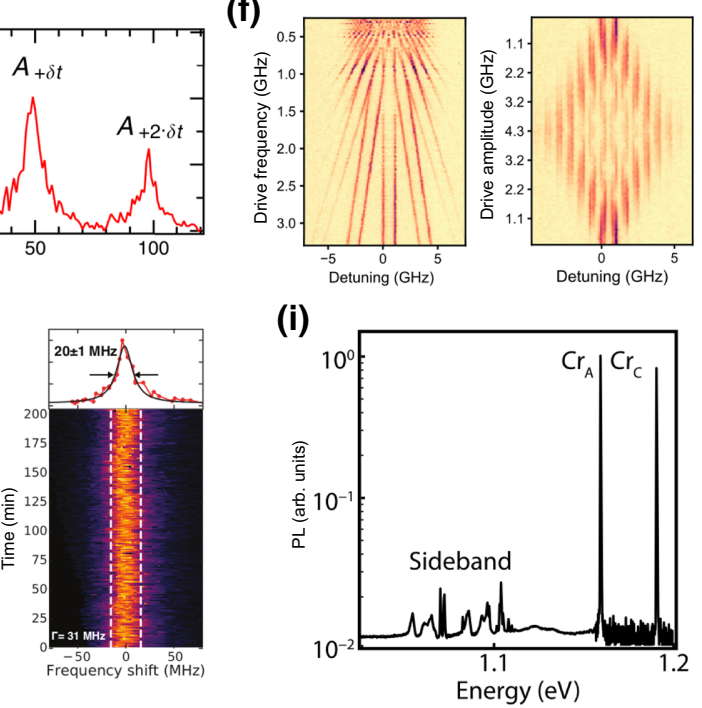

(i)

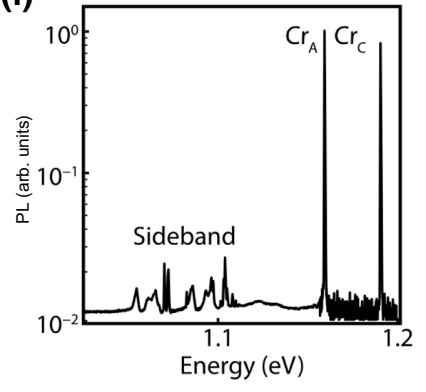

FIG. 2. Optically active spins in SiC. (a) The 4H-SiC crystal lattice, showing the inequivalent configurations of the divacancy, silicon vacancy $\left(\mathrm{V}_{\mathrm{Si}}\right)$, and chromium ion. (b) Patterning of $\mathrm{V}_{\mathrm{Si}}$ using a focused proton beam enables three-dimensional control over placement of single defects and ensembles. (c) Direct laser writing of single $\mathrm{V}_{\mathrm{Si}}$ for on-demand color-center generation in SiC devices under test. (d) Stable, nearly lifetime-limited optical emission from the $\mathrm{V}_{\mathrm{Si}}$ under above-resonant excitation. (e) The demonstration of indistinguishable single-photon emission from the $\mathrm{V}_{\mathrm{Si}}$. (f) The $\mathrm{V}_{\mathrm{Si}}$ remains spectrally stable even under high-amplitude, fast Stark shift modulation, enabling the observation of optical Floquet eigenstates. (g) Advanced epitaxy techniques available commercially for SiC made possible the integration of high-quality divacancy color centers into $p-i-n$ junctions, enabling the demonstration of nearly $1 \mathrm{THz}$ of Stark shift. (h) By depleting the charge-trap environment via reverse bias in the $p$ - $i$ - $n$ junction, linewidth narrowing from $1 \mathrm{GHz}$ to $20 \mathrm{MHz}$ is observed, approaching the transform limit. (i) Among the nascent optically addressable defects in SiC, the chromium ion stands out for, among other characteristics, its high Debye-Waller factor of 75\%. Reproduced from (b) Ref. [73], (c) Ref. [74], (d), (e) Ref. [52], (f) Ref. [54], (g),(h) Ref. [63], (i) Ref. [70]. 
of $200 \mathrm{GHz}$ [54] is sufficient to overcome spectral inhomogeneity of defect ensembles [35], a prerequisite for spin-spin entanglement of multiple $\mathrm{V}_{\mathrm{Si}}$ via photon interference. Furthermore, owing to the remarkable spectral stability of the $\mathrm{V}_{\mathrm{Si}}$ optical transitions, spectrally reconfigurable single-photon emission from the $\mathrm{V}_{\mathrm{Si}}$ has been obtained via Floquet engineering [54] [Fig. 2(f)]. Integration into semiconductor devices such as the $p-i-n$ junction has enabled electrical readout of the spin state [86] and electrical control of the charge state [87] at room temperature. An essential next step toward realizing useful quantum photonic devices with the $\mathrm{V}_{\mathrm{Si}}$ will be the demonstration of spectrally stable near-transform-limited defects in nanostructures.

\section{The divacancy}

The neutral divacancy is composed of adjacent silicon and carbon vacancies, denoted by $\mathrm{V}_{\mathrm{Si}} \mathrm{V}_{\mathrm{C}}^{0}$. The combination of $C_{3 v}$ symmetry, six active electrons, and spin-1 electronic structure render the optical and spin properties of the defect similar to the nitrogen-vacancy (NV) center in diamond. However, the optical transitions are in the $1100 \mathrm{~nm}$ range, which is more favorable than the diamond NV centers 637 $\mathrm{nm}$ emission for optical communications and for integration into nanophotonic structures; The DWF of 0.07 [61] is also an improvement over the NV center. Single divacancies with narrow optical linewidths and spin coherence up to $1 \mathrm{~ms}$ have been observed in $\mathrm{SiC}$ crystals without isotope purification [61]. Recently, the discovery of dressed clock transitions have enabled the demonstration of divacancy coherence of $64 \mathrm{~ms}$ in material with natural isotope content [11]. Divacancy ensembles have been used to achieve a high degree of polarization of the $\mathrm{SiC}$ nuclear spin bath $[58,60]$. Ensemble entanglement with nuclear spins at ambient conditions has been shown [59], as well as the control of single divacancy-coupled nuclear spins [88]. Electrical and mechanical spin control of the divacancy have been demonstrated [89-91]. In a major step toward wafer-scale optical and electrical integration of color centers, commercial $p-i-n$ junction $\mathrm{SiC}$ devices have been engineered to host individually addressable divacancies with nearly lifetime-limited optical transitions, millisecond spin-coherence times, as well as optical and electrical charge control [63]. Furthermore, these devices can produce a Stark shift as large as $850 \mathrm{GHz}$ [Figs. 2(g) and 2(h)]. This is the first demonstration of such a combination of state-of-the-art optically addressable spin-qubit properties in a single scalable semiconductor platform, opening opportunities for multiqubit integration once combined with LDOS-enhancing photonic structures.

\section{Emerging defects}

Numerous other defects in $\mathrm{SiC}$ are currently under investigation for applications in quantum photonics. The nitrogen-vacancy center $\left(\mathrm{N}_{\mathrm{C}} \mathrm{V}_{\mathrm{Si}}^{-}\right)$in $\mathrm{SiC}$ has been identified as a color center with a favorable emission frequency near the telecommunications S-band [64-67]. Recently, coherent spin control has been observed in $\mathrm{N}_{\mathrm{C}} \mathrm{V}_{\mathrm{Si}}^{-}$ensembles [68]. Further studies are necessary to investigate the cause of the low brightness of the $\mathrm{N}_{\mathrm{C}} \mathrm{V}_{\mathrm{Si}}^{-}$as compared to its diamond counterpart; it may be due to a low quantum efficiency (i.e., a large percentage of the excited state decay is into the nonradiative intersystem crossing) or a long-lived metastable state. Another defect, the chromium ion $\left(\mathrm{Cr}^{4+}\right)$, has been identified as a promising quantum memory. $\mathrm{Cr}^{4+}$ has an optical excited state lifetime of 155 $\mu \mathrm{s}$, and emits $75 \%$ of photons into the ZPL in the nearIR [see Fig. 2(i)] [69,70]. This suggests that integration of the $\mathrm{Cr}^{4+}$ into nanophotonic structures may enable a large reduction of lifetime, which is essential for the efficient readout of single spins without a cycling transition [26]. However, the intrinsic optical and spin coherence of $\mathrm{Cr}^{4+}$ defects remains an outstanding question; so far, only high density $\mathrm{Cr}^{4+}$ ensembles have been studied, where the optical linewidths are $10^{4}$ times broader than lifetime limited, and the measured spin-coherence time of $81 \mu \mathrm{s}$ is limited by spin-spin interactions in the ensemble [70]. Finally, we highlight the vanadium impurity in $\mathrm{SiC}, \mathrm{V}^{4+}$, which is notable for its emission in the O-band and unusual optical lifetime of 108 and $167 \mathrm{~ns}$ (for the brighter inequivalent lattice sites) [72]. Particularly interesting is the strong sensitivity of the $\mathrm{V}^{4+}$ optical transition to nearby nuclear spins, suggesting potential applications for optically resolved nuclear spin registers [72].

\section{B. SiC photonics}

Wafer-scale growth and processing of $4 \mathrm{H}$ and $6 \mathrm{H}$ polytypes of $\mathrm{SiC}$ was developed in the 1990s for applications in high-power electronics. Soon after, $4 \mathrm{H}$ - and $6 \mathrm{H}-\mathrm{SiC}-\mathrm{on}-$ insulator (SiCOI) were demonstrated [92] using the same ion-implantation (Smart-Cut) method that is used to produce silicon-on-insulator (SOI) wafers. This technology enabled the first demonstration of photonic crystal cavities in $\mathrm{SiC}$ (Fig. 3) [93,94]. As the development of photonics in Smart-Cut 4H-SiC continued [95] and Smart-Cut SiCOI became optimized on a wafer scale [96], the intrinsic optical absorption of the $\mathrm{SiC}$ thin films was identified as the limiting factor for high- $Q$ SiC photonics, limiting waveguide losses to $>5 \mathrm{~dB} / \mathrm{cm}$ [97]. Although further optimization of the implantation conditions may remedy the low material quality [95], it is unclear whether the Smart-Cut method is suitable for producing films of $\mathrm{SiC}$ with the same nearly pristine crystal quality as siliconon-insulator. The difference between Smart-Cut SOI and SiCOI stems from the drastically different thermal properties of silicon and SiC: the lattice of silicon will soften and heal at the modest temperatures achievable in standard 


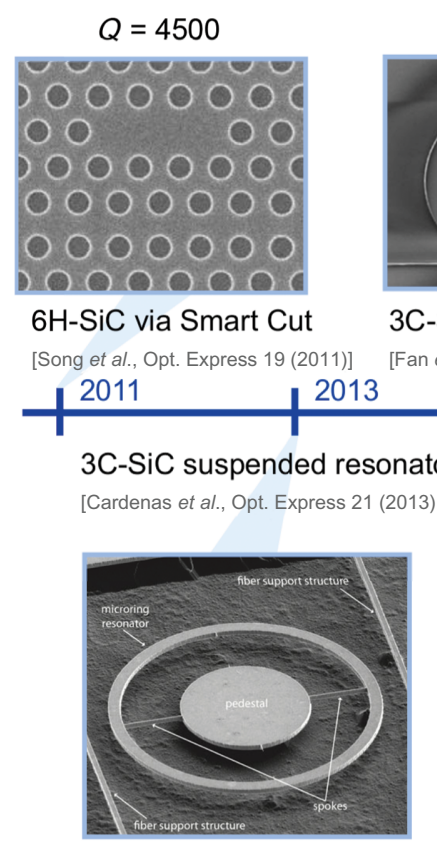

$Q=1.4 \times 10^{4}$
$Q=2.4 \times 10^{5}$

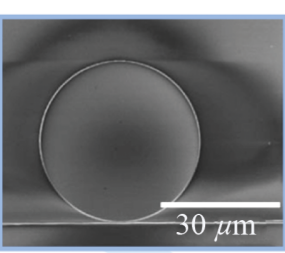

$3 \mathrm{C}-\mathrm{SiC}$ on insulator

Fan et al., Opt. Lett. 45 (2020)] 2019

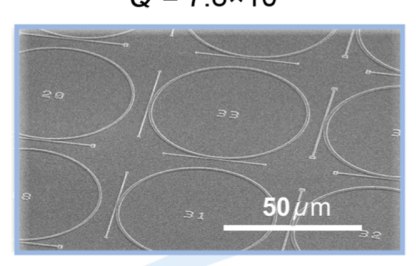

$4 \mathrm{H}-\mathrm{SiC}$ on insulator

[Lukin et al., Nat. Photonics 14 (2019)]
Efficient second harmonic generation in ultra-high $\mathrm{Q} 4 \mathrm{H}-\mathrm{SiC}$ cavities [Song et al., Optica 6 (2019)]

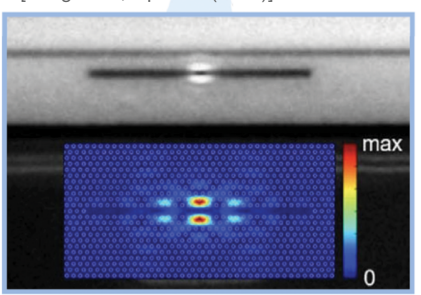

$Q=6.3 \times 10^{5}$

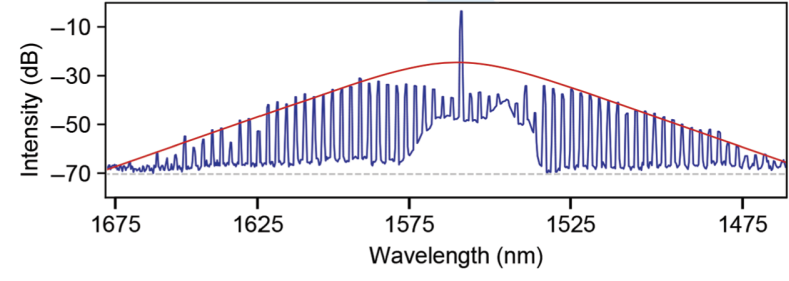

FIG. 3. Timeline of SiC photonics development. First demonstration of a SiC photonic device using the Smart-Cut approach with $6 \mathrm{H}-\mathrm{SiC}$ [93]. Soon after, suspended resonators in 3C-SiC-on-Si were demonstrated [98]. Strong intrinsic absorption of low-quality Smart Cut and heteroepitaxial 3C films was hypothesized to limit the achievable $Q$ factors. Using thicker 3C-SiC epilayers or thinning down bulk-crystal 4H-SiC, enabled record $Q$ factors in 3C-SiC [101,102], ultra-high $Q$ photonic crystal cavities [33], and low-loss $4 \mathrm{H}-\mathrm{SiC}$-on-insulator waveguides [32]. Recently, devices with $Q$ factors exceeding $10^{6}$ were shown, enabling the demonstration of optical parametric oscillation and microcomb formation [34]. Reproduced from Refs. [32-34,93,98,102].

quartz furnaces. $\mathrm{SiC}$, in turn, is one of the most refractory materials, subliming at $2700^{\circ} \mathrm{C}$. Repairing the lattice in postprocessing without destroying the substrate is thus likely impossible.

Another approach to $\mathrm{SiC}$ photonics took advantage of the heteroepitaxial growth of $3 \mathrm{C}-\mathrm{SiC}$ films on silicon. A variety of $3 \mathrm{C}$-SiC-on-Si photonic devices have been demonstrated, including photonic crystal cavities $[98,99]$ and whispering-gallery-mode resonators [100]. However, this approach also suffers from substantial intrinsic material absorption, due to the high density of crystal defects near the growth interface caused by the $\mathrm{Si}-\mathrm{SiC}$ lattice mismatch. Recently, a technique based on film transfer and back-side polishing introduced the 3C-SiC-on-insulator platform and enabled waveguides with losses down to $1.5 \mathrm{~dB} / \mathrm{cm}$, still likely limited by material absorption $[101,102]$.

It is in the quantum context that the material quality of $\mathrm{SiC}$ thin films falls under the highest scrutiny: the coherence properties of color centers are highly sensitive even to low densities of unwanted defects. Indeed, in the first demonstration of $\mathrm{SiC}$ color centers coupled to a nanophotonic resonator - using photonic crystal cavities fabricated in $3 \mathrm{C}-\mathrm{SiC}$-on- $\mathrm{Si}$ - the color-center optical coherence was shown to be severely degraded by the lattice mismatch between $\mathrm{Si}$ and $\mathrm{SiC}$ [103]. Similarly, color centers with good optical coherence have not been observed in SmartCut SiCOI, a consequence of the lattice damage induced by Smart-Cut ion implantation: the dose required in the Smart-Cut process $\left(10^{16}-10^{17}\right.$ ions $\left./ \mathrm{cm}^{2}\right)$ exceeds the dose used to generate spatially resolvable single defects by 4 orders of magnitude. Incidentally, similar challenges arose in the development of thin-film diamond quantum photonics. In diamond, the absence of high-quality thin films prompted the development of bulk-carving methods, such as angle etching [104], for fabricating nanophotonic devices. The same technique has recently been demonstrated in $\mathrm{SiC}$ [105]. An alternative bulk-carving technique has been developed for $4 \mathrm{H}-\mathrm{SiC}$, relying on the advanced $\mathrm{SiC}$ homoepitaxy technology and doping-selective photoelectrochemical etching [106]. This method enabled the first demonstration of Purcell enhancement of emission from $\mathrm{V}_{\mathrm{Si}}$ ensembles in $4 \mathrm{H}-\mathrm{SiC}$ photonic crystal cavities [106]. Recently, using the same photonics platform, coherent spin control of a single divacancy integrated into photonic crystal cavities was realized [107] [Figs. 4(c)-4(e)].

Leveraging the wafer-scale production of $4 \mathrm{H}-\mathrm{SiC}$ and the advanced grinding and polishing equipment developed for processing it, a method for fabricating "quantumgrade" 4H-SiC-on-insulator was recently introduced [32]. This method enables $4 \mathrm{H}-\mathrm{SiCOI}$ substrates with the same crystalline quality as bulk $\mathrm{SiC}$ crystal. Using $4 \mathrm{H}-\mathrm{SiCOI}$ 
(a)
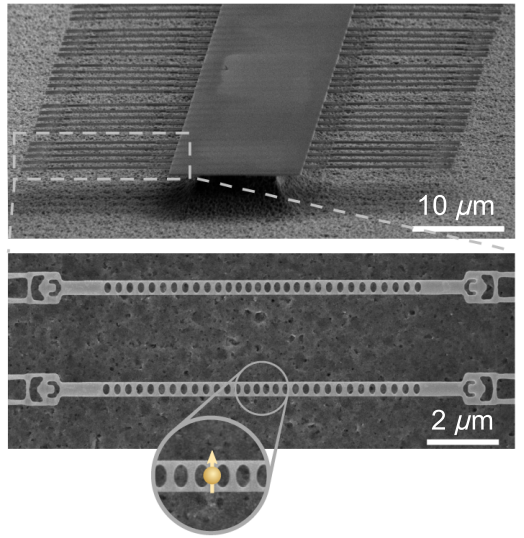

(c)

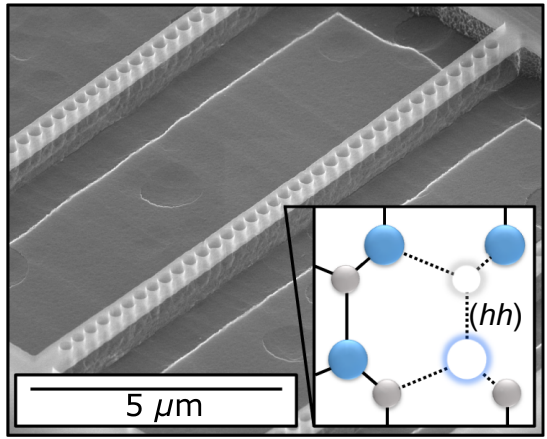

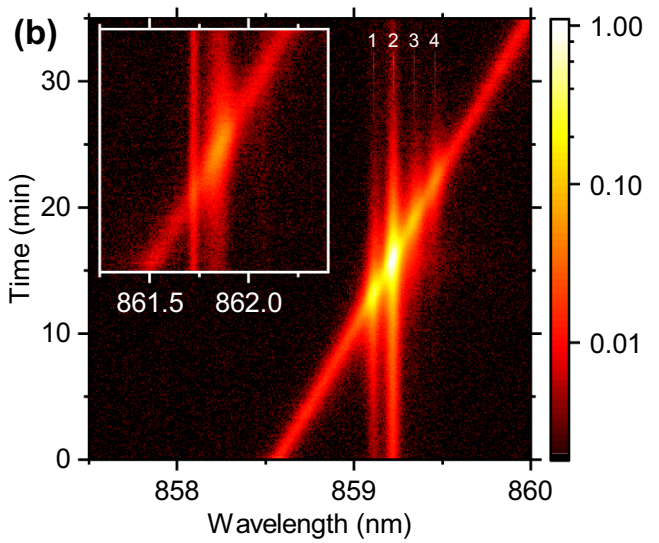

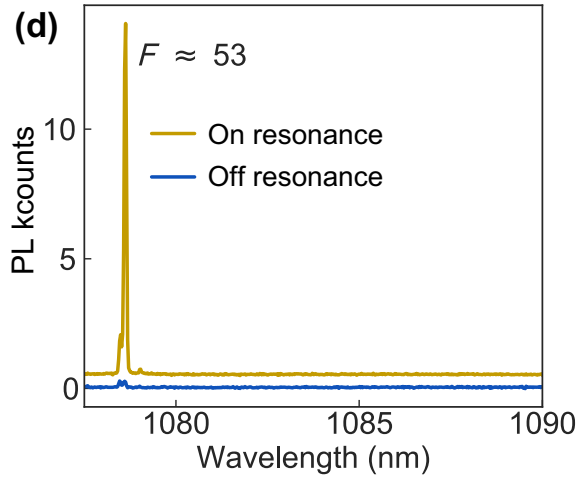

(e)

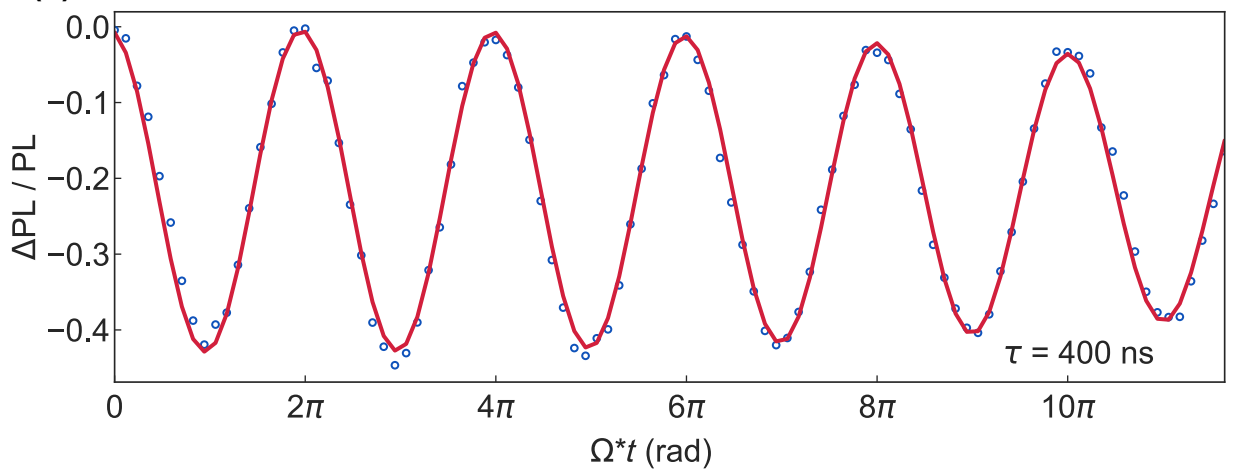

FIG. 4. SiC quantum photonics with defects. (a) An array of nanophotonic cavities fabricated in $4 \mathrm{H}-\mathrm{SiC}$-on-insulator, integrated with single $\mathrm{V}_{\mathrm{Si}}$ color centers [32]. (b) A cavity is tuned via gas condensation through the color-center resonance, thereby enhancing the optical transition by 120 times. (c) Nanophotonic cavities fabricated via the photoelectrochemical undercut technique [107], integrated with single divacancies. (d) Divacancy emission spectrum on and off resonance with the cavity, showing enhancement of 53 times. (e) Coherent spin control of the cavity-integrated divacancy. Reproduced from (a),(b) Ref. [32], (c)-(e) Ref. [107]. produced this way, ultra-high-quality $(Q)$ factor photonic crystal cavities $\left(Q=6.3 \times 10^{5}\right)$ have been fabricated [33]. $4 \mathrm{H}-\mathrm{SiCOI}$ also enabled quantum photonic devices with single color centers in a CMOS-compatible architecture [32] [Figs. 4(a)-4(b)]. Unfettered by the material absorption limit of previous approaches, integrated $\mathrm{SiC}$ photonics with propagation loss below $0.5 \mathrm{~dB} / \mathrm{cm}$ (corresponding to a $Q$ factor exceeding 1 million) have become possible. Lowloss microring resonators were used to demonstrate optical parametric oscillation and microcomb formation [34], establishing $\mathrm{SiC}$ as a promising material for integrated nonlinear photonics. Recently, the intrinsic absorption of $4 \mathrm{H}-\mathrm{SiC}$ was measured to be as low as $0.02 \mathrm{~dB} / \mathrm{cm}$ in an unoptimized sublimation-grown sample [34], suggesting that integrated photonics in $\mathrm{SiC}$ with $\mathrm{Q}$ factors of at least $10^{7}$ are possible.

\section{Comparison with diamond quantum photonics}

The key color-center quantum photonic demonstrations (such as single-shot readout of spin [108,109], cavity integration of emitters with narrow optical transitions [21,110,111], cavity-mediated spin-spin interactions [24], and nuclear spin quantum register [10]) have so far been in the diamond platform, specifically with the NV center and the silicon-vacancy color center. Both of these color centers possess at least one optical transition that does not suffer from nonradiative spin-flip processes. The cyclicity of such a transition was utilized for single-shot readout of spin, even without photonic cavity integration [108,109]. A similar cycling transition has not yet been definitively demonstrated in a $\mathrm{SiC}$ color center, although a potential candidate in the $k h$ divacancy has been identified [62]. However, a cycling transition is not required 
for single-shot readout if cyclicity can be enhanced with a photonic cavity via the Purcell effect [26]. So far, only centrosymmetric color centers in diamond have been integrated into nanophotonic structures while retaining narrow linewidths [111-113]. Although inversion symmetry is not in principle a prerequisite for defect insensitivity to electric fields [85], integration of a noncentrosymmetric defect into a nanostructure while preserving its spectral stability is yet to be demonstrated. It should be noted that crystals without inversion symmetry such as $\mathrm{SiC}$ do not host inversion-symmetric defects.

With regard to photonic devices, state-of-the-art microring resonators in $\mathrm{SiC}$ and diamond are currently comparable $[34,114]$, whereas the photonic crystal nanocavities in $\mathrm{SiC}$ are superior due to the wider range of device designs accessible in the thin-film platform [33,104]. There are some key differences between diamond and $\mathrm{SiC}$ with regard to the intrinsic material properties. The larger bandgap of diamond results in a wider transparency window into the ultraviolet range. Although the $\mathrm{SiC}$ bandgap is narrower, it strikes a balance between optical transparency (which spans the visible frequencies) and ease of doping, enabling semiconductor structures such as $p-i-n$ junctions. The $\mathrm{SiC} \chi^{(3)}$ nonlinearity is an order of magnitude stronger than that of diamond, lowering the power requirement for generating optical parametric oscillation and optical frequency combs $[34,114]$. SiC also possesses a strong $\chi^{(2)}$ nonlinearity of $12 \mathrm{pm} / \mathrm{V}$ [115], which is absent in diamond due to the inversion symmetry of its lattice.

\section{TOWARD MULTINODE QUANTUM PHOTONICS WITH DEFECTS}

\section{A. Optimizing the single quantum node \\ 1. Emitter-cavity coupling}

As motivated in the Introduction, the density of states of a defect's electromagnetic environment must be modified in order to enhance and direct its photon emission, suggesting a nanophotonic cavity coupled to an optically active spin qubit as the basic building block of a defect-based integrated quantum photonic device. Despite remarkable progress in the development of the cavity-defect node, there remains a large performance gap between integrated quantum photonic devices and their classical photonic counterparts. To date, the state-of-the-art photonic crystal cavities in silicon [116] and whispering gallery resonators in silica [117] have $Q$ factors of $10^{7}$ and $10^{9}\left(10^{8}\right.$ for a fully integrated device [118]), respectively. In contrast, photonic devices integrated with spin defects have so far demonstrated $Q$ factors in the $10^{3}-10^{4}$ range. It is not surprising that spin-defect photonics are orders of magnitude behind, since the quantum material platforms (i.e., diamond, YSO, YVO, YAG, and $\mathrm{SiC}$ ) are relatively new to the photonics scene. From this perspective, $\mathrm{SiC}$-on-insulator is uniquely suited for bridging the classical-quantum photonics gap. SiCOI is already in the top three platforms for demonstrating high $Q / V$ photonic resonators, after silicon-oninsulator [116] and lithium niobate-on-insulator [119]. The high refractive index of $\operatorname{SiC}(n=2.6)$ allows for the fabrication of resonators based on two-dimensional photonic crystal cavities, the class of nanophotonic devices with the highest $Q / V$ ratio to date [116]. The impact of bridging the classical-quantum photonics gap will be an orders-of-magnitude improvement in readout fidelity and entanglement generation rates over current state-of-the-art spin-qubit photonic demonstrations. Furthermore, it will unlock new regimes of spin-qubit operation, including generation of transform-limited (and thus indistinguishable) photons from rare-earth ions [25] and strong coupling of a color center to a cavity [21]. In $\mathrm{SiC}$, these advances could likely be implemented with defects such as the $\mathrm{Cr}^{4+}$ ion, the divacancy, and the silicon vacancy.

What $Q / V$ is sufficient, then? Naturally, the answer will depend on the defect used and the application. A defect with a lower branching ratio into the enhanced transition (for example, due to a small DWF or low quantum efficiency) will require greater $Q / V$ to achieve the same Purcell enhancement. From the photonics perspective, the emitter-cavity node is sufficiently optimized once the fraction of emitter excitations that do not result in a photon emitted into the cavity becomes negligible compared to other system losses, and the spin state readout fidelity exceeds the fidelity of other single-register qubit operations. For readout, the presence of a cycling transition would relax the $Q / V$ requirements [21,26]. In selecting a promising quantum emitter, the brightness of a defect plays a role. High brightness can be an indicator of high quantum efficiency and the presence of cycling transitions. Low brightness, however, does not necessarily mean the contrary, since the presence of a long-lived metastable state can result in low count rates even if the nonradiative decay rate into the metastable states is slow. Thus, in contrast with single-photon source applications, the defect brightness is not the key metric in spin-based quantum technologies.

\section{Mitigating emitter degradation in nanostructures}

Another challenge for defect-based quantum photonics is mitigating the degradation of optical properties of defects in nanostructures. Often, defects with narrow, stable transitions in bulk material degrade severely when a material interface is nearby. The common understanding is that the linewidth degradation is caused by spectral diffusion (i.e., rapid temporal fluctuations in the defect's optical transitions due to fluctuating charges on the nearby surfaces). So far, the greatest success in nanophotonic integration has been had with centrosymmetric defects in 
diamond such as the silicon vacancy $[21,111,112]$, the germanium vacancy [112], and the tin vacancy [113]. And yet, even these defects that should be maximally insensitive to environmental fluctuations display spectral diffusion that by several times exceeds the transform-limited linewidth, likely as a result of strain introduced during growth and fabrication, which lifts the symmetry of these defects and renders them sensitive to the electric field to first order [111-113]. The problem of spectral stability must be solved before the spin-defect quantum photonics technology becomes scalable.

Unlike many intrinsic properties of defects over which the experimentalist has no control, spectral diffusion in nanostructures is an extrinsic property and is amenable to systematic material science engineering. Surface passivation, either chemical or plasma assisted, is one technique that has been successfully used to increase the photon or phonon lifetime in nanoresonators [120,121], but has not yet been explored for defect stabilization. A comprehensive study that seeks to understand rather than simply eliminate spectral diffusion is needed to resolve this problem globally. For example, a comparison of the spectral diffusion of different defect types in the same environment can help elucidate the degradation mechanisms.

The approach toward understanding spectral diffusion and resolving it will likely be specific to each quantum material platform. In the case of $\mathrm{SiC}$, surfaces may potentially be passivated with a graphene layer, which can be readily grown on hexagonal $\mathrm{SiC}$ [122]. Although the strong optical absorption of graphene renders this an impractical solution when combined with photonics, it would constitute a valuable proof-of-concept demonstration of passivation-enabled compensation of spectral diffusion. An entirely different method to achieve nearsurface emitter stabilization in SiC may take advantage of charge depletion using advanced doping epitaxy available in SiC [63]; implemented successfully in bulk material, the charge depletion technique has yet to be investigated in nanostructures. We note here that charge depletion can be directly integrated with existing nanophotonic architectures, as has been done in other platforms [123]. Although the high temperature required for dopant activation in $\mathrm{SiC}$ [124] would necessitate definition of the diode structure prior to the fabrication of the SiCOI material stack [32], it is a technologically straightforward process. Another active approach to mitigating the effects of spectral diffusion may be via optimized optical excitation of the emitter, either via time-dependent drive [125] analogous to radio-frequency modulation demonstrations to extend defect spin coherence [126], or via optimized steadystate illumination [127]. Fortunately, the challenge of stabilization of defects in nanostructures is as formidable as the possible strategies for overcoming it are numerous.

\section{B. Scaling-up quantum photonic processors}

When designing a nanophotonic defect-based quantum node, it is crucial to look ahead toward multinode scalability. This introduces two additional single-node system requirements. First, since the nodes must be spectrally identical during operation, the single node must be spectrally tunable to overcome inherent variations in resonator frequencies and the inhomogeneous broadening of defects. Second, the node must have an efficient waveguide interface, in order to transfer the emitted photons into the internode link with very high efficiency. The exact efficiency requirements will depend strongly on the application. Quantum communication and simulation will likely place a less stringent requirement than fault-tolerant quantum computation, where proposals require no more than $10 \%$ cumulative loss at all stages of the circuit $[14,15]$.

The spectral tuning of cavities and color centers has seen excellent progress in a variety of platforms. For cavity tuning, the primary technique has been cryogenic gas condensation [24]: a heated gas tube delivers argon or xenon gas to the sample that then condenses on the cold sample surface, red shifting the cavity frequency. Since condensed gas can be selectively and gradually removed via heating by a milliwatt laser, one can tune individual cavities onto resonance by applying the appropriate laser pulse. Numerous other techniques have also been employed, including atomic layer deposition [128], laser-assisted oxidation [129], and index-shifting materials [130]. In all, cavity tuning is amenable to further optimization and numerous routes to multinode scalability exist. We note that, although in principle the electro-optic effect allows fast modulation of cavity resonance, the Stark effect in emitters is typically much stronger and thus if rapid modulation is desired, it will likely be advantageous to modulate the emitter with respect to the cavity rather than vice versa. Defect tuning has been demonstrated using both Stark shift $[32,63]$ and strain [131], with several demonstrations of the tuning range far exceeding the inhomogeneous broadening. Fast, high-amplitude spectral modulation of quantum emitters has been shown [54,62]. One caveat, however, is that current tuning demonstrations are limited to compensating one degree of freedom, whereas spectral inhomogeneity in defects is higher dimensional in nature (a vector for the electric field and a tensor for the strain). Thus, for most defects (an exception are defects with degeneracy broken by spin-spin interactions only, like the $\mathrm{V}_{\mathrm{Si}}$ [83]), a single tuning degree of freedom is only sufficient to make one optical transition degenerate [131]. Thus, if a protocol makes use of multiple optical transitions of a single defect, each additional transition must be controlled with an independent tunable laser, which is not scalable [111]. Cavity-assisted Raman emission can allow us to overcome this limitation [132]. Overall, while engineering challenges are still ahead, there is already a framework for building 
a fully tunable quantum node and a clear path toward scalability.

In addition to spectral tuning, an efficient cavitywaveguide interface is the second essential requirement for multinode devices. To achieve this, the cavity must be designed to be significantly over coupled to the waveguide (i.e., cavity loss into the waveguide must dominate over other loss channels), implying that ultimately the actual $Q$ factor will be much lower than the highest $Q$ attainable in the platform. Consequently, this places an even greater requirement on the intrinsic cavity $Q / V$ to maintain the same Purcell enhancement. Furthermore, once the photon has entered the waveguide, great attention must be paid to any losses that the photon may experience as it propagates between nodes.

At this point, it is important to note a distinction between the principles of scalability for classical computers and quantum photonic processors. Classical technologies based on semiconductor transistors progressed through "scaling down," where the individual node size has been reduced further and further to accommodate greater computing power, until the limiting factor has become the wires rather than the transistors themselves. In contrast, a defect-based quantum photonic computer does not fundamentally enjoy gains from small overall size (as long as the photon emission-enhancing component maximizes the large Purcell factor through high $Q / V$ ). Compared to the resistive losses that limit classical computers, short-distance optical communications (fibers or waveguides) are effectively lossless. The speed of a defect-based quantum computer will not be limited by the internodelink communication rates (photon transit time), but by the (much slower) physics of the quantum node, namely, the spin manipulation and readout of the defect-cavity system. This difference in paradigms is especially relevant in context of the distinct challenge of device yield present in defect-based quantum photonics, since the technological complexity of the single node precludes fabricating quantum nodes with a yield exceeding $99 \%$. This suggests that scaling up will require a degree of device postselection and reconfigurability, as is already done on other platforms such as trapped atoms [136]. In light of the above considerations, integration via off-chip fiber interconnects should not a priori be excluded in the near future (a)
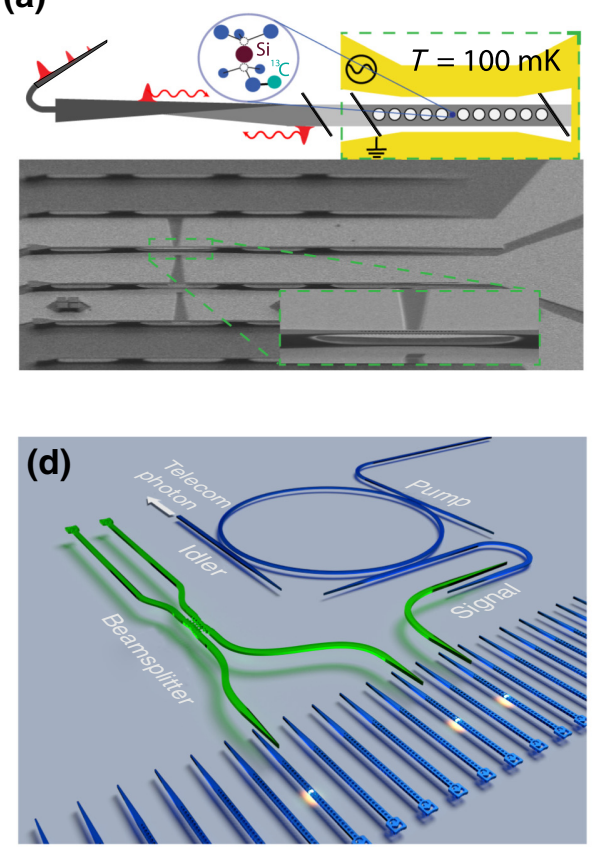

(b)

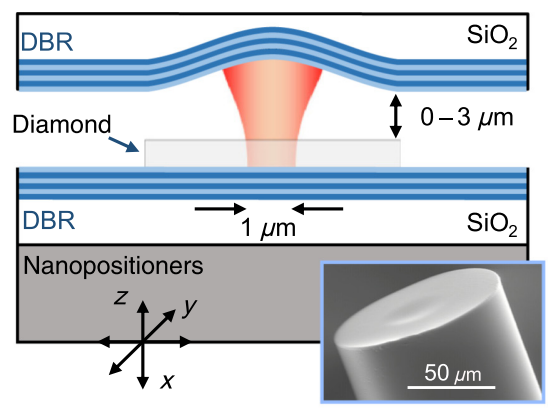

(c)

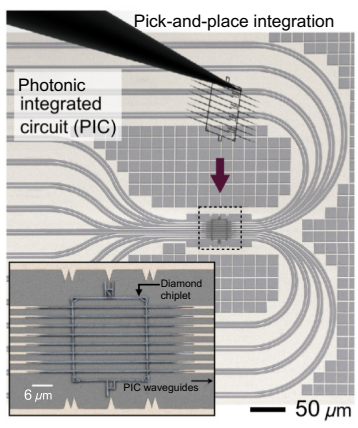

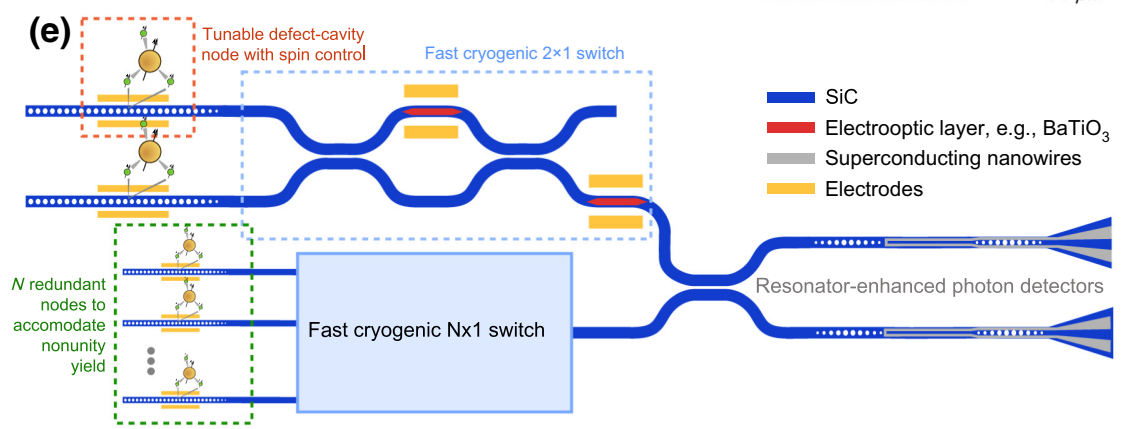

FIG. 5. Approaches to scaling-up spin-based quantum photonic technologies. (a) Diamond nanophotonic cavity with a single siliconvacancy defect and an adiabatically coupled fiber interface [133]. (b) Free-space coupled Fabry-Pérot microcavity enhancing the emission of a NV center in diamond [134]. Inset: the concave mirror can also be fabricated directly on the tip of a fiber [135]. (c) Pick-and-place heterogeneous on-chip integration of diamond microchiplets containing silicon- and germanium-vacancy centers on top of aluminum nitride photonic waveguides [112] (image courtesy of Noel Wan). (d) A heterogeneous approach without pick-andplace can be realized by using a secondary layer of photonic interconnects to postselect working quantum nodes [32]. (e) A conceptual diagram demonstrating how the example photonic network shown in Fig. 1(b) could be realized in a fully monolithic platform. In order to account for nonunity fabrication yield, $N$ redundant nodes are fabricated in the place of one node, and a $N \times 1$ switch (composed of cascaded $2 \times 1$ switches) selects one working node. Reproduced from (a) Ref. [133], (b) Refs. [134,135] (d) Ref. [32]. 
(before the increasing number of quantum nodes makes it impractical).

In this context, we comment on the current leading approaches toward multinode scalability.

\section{Integrated nanophotonic devices with a fiber interface}

This approach relies on a nanophotonic cavity for enhancing the light-matter interaction, but routes the photons from the cavity directly into a fiber for off-chip processing [Fig. 5(a)] [21,110,111]. This approach naturally enables $100 \%$ device yield within a quantum network via postselection: individual devices are characterized and working devices are integrated together via (low-loss) fiber interconnects. Because the nanophotonic waveguide and optical fibers are effectively lossless at the relevant length scales, photon loss is incurred exclusively at the fiber-waveguide interface, with demonstrated efficiencies as high as $96 \%$ [110].

\section{Fabry-Pérot microcavities}

Recently, photonic resonators based on concave dielectric mirrors have enabled breakthrough demonstrations of cavity-integrated light-matter interactions [137]. In this approach, a concave mirror is fabricated [138] either in bulk silica [134] or, notably, directly on the tip of a fiber [135], and forms a distributed Bragg reflector microcavity with the buried Bragg mirror beneath the active quantum medium, incorporating the defect in between [Fig. 5(b)]. As with fiber-coupled on-chip nanophotonic cavities, unity yield is achieved by postselection; the fiber-tip scans the surface to isolate a suitable defect, and the cavity resonance is tuned by controlling the fiber height piezoelectrically. Notably, this technique can in principle be applied to any defect that can be integrated into a smooth, thin membrane $[134,139,140]$. The fiber Fabry-Pérot microcavity is in a sense a distillation of the fiber-coupled nanophotonic cavity approach, as the cavity output mode is Gaussian and can efficiently be coupled into single-mode fibers. The efficiency is limited by losses in the optical components required for coupling the photons into the single-mode fiber, reflection losses at surfaces, and a slight modal mismatch between the cavity and fiber modes. Recently, a total coupling efficiency of $68 \%$ into the fiber mode has been demonstrated [141].

\section{Pick-and-place heterogeneous on-chip integration}

This approach aims to address the concern of yield in a fully integrated fashion, by transferring quantum nodes onto an integrated photonic circuit after characterization. Pick and place is a particularly promising technique in material platforms not suitable for standard photonics processing: for instance, it has recently enabled large-scale on-chip integration of diamond color centers with aluminum nitride interconnects [Fig. 5(c)] [112]. Although promising for fully chip-scale integration, pick and place currently suffers from high experimental losses in the adiabatic transfer of photons from the transferred quantum material to the integrated photonic circuit (transmission of $34 \%$ [112]), suggesting that the transfer efficiency to the internode link may be a serious impediment for scalability. Although highly efficient interlayer adiabatic transfer is possible (approaching 99\% [142]), it relies on long, well-aligned $(200 \mu \mathrm{m})$ tapers that are difficult to achieve using a pick-and-place technique [but are possible with heterogeneous integration of multiple thin-film layers; see Fig. 5(d)]. Thus, we see an efficient waveguide interface as the key challenge in developing pick and place as a method for multinode QIP.

\section{PERSPECTIVE ON FULLY INTEGRATED QUANTUM PHOTONICS}

Most advanced spin-defect experiments to date rely on the strategy of coupling emitted photons into an optical fiber as soon as possible (approaches 1 and 2 above). However, for applications other than fiber network communications, a photon in a fiber is not an advantage over a photon in an integrated waveguide: practical realization of the key components for quantum networks identified in Fig. 1 actually plays to the strengths of integrated photonics rather than fiber optics. Integrated photonics have already achieved system complexity beyond what can be practically realized in macroscopic fiber-based devices, integrating hundreds of elements with mean fidelities of linear components exceeding 99.9\% [4]. On-chip integration of single-photon detectors has seen remarkable progress in recent years $[38,143,144]$, and integration with photonic resonators will likely enable narrowband integrated single-photon detection with efficiencies exceeding $99 \%$ in the near future. In our view, the chip-integrated approach is the most promising for large-scale quantum systems. This architecture is illustrated in Fig. 5(e), using the example photonic network introduced in Fig. 1(b).

In this fully monolithic realization of chip-integrated spin-based quantum technologies, the photon never leaves the chip, never couples into a fiber or passes through lossy bulk active elements, and is not subject to system fluctuations inherent in a large-scale macroscopic system. In fact, the photon never leaves the waveguide into which the quantum node emitted it, because switching, interference, and detection can all be realized in a waveguide geometry $[4,144]$. Compact and efficient on-chip photon detectors can be placed anywhere in the integrated circuit to convert a (fragile) quantum signal into a (robust) classical signal that can be routed off chip via standard CMOS electronics such as vias and buried electrical layers, aiding in the realization of circuit connectivity. The relative simplicity of the integrated approach is a source of optimism for 

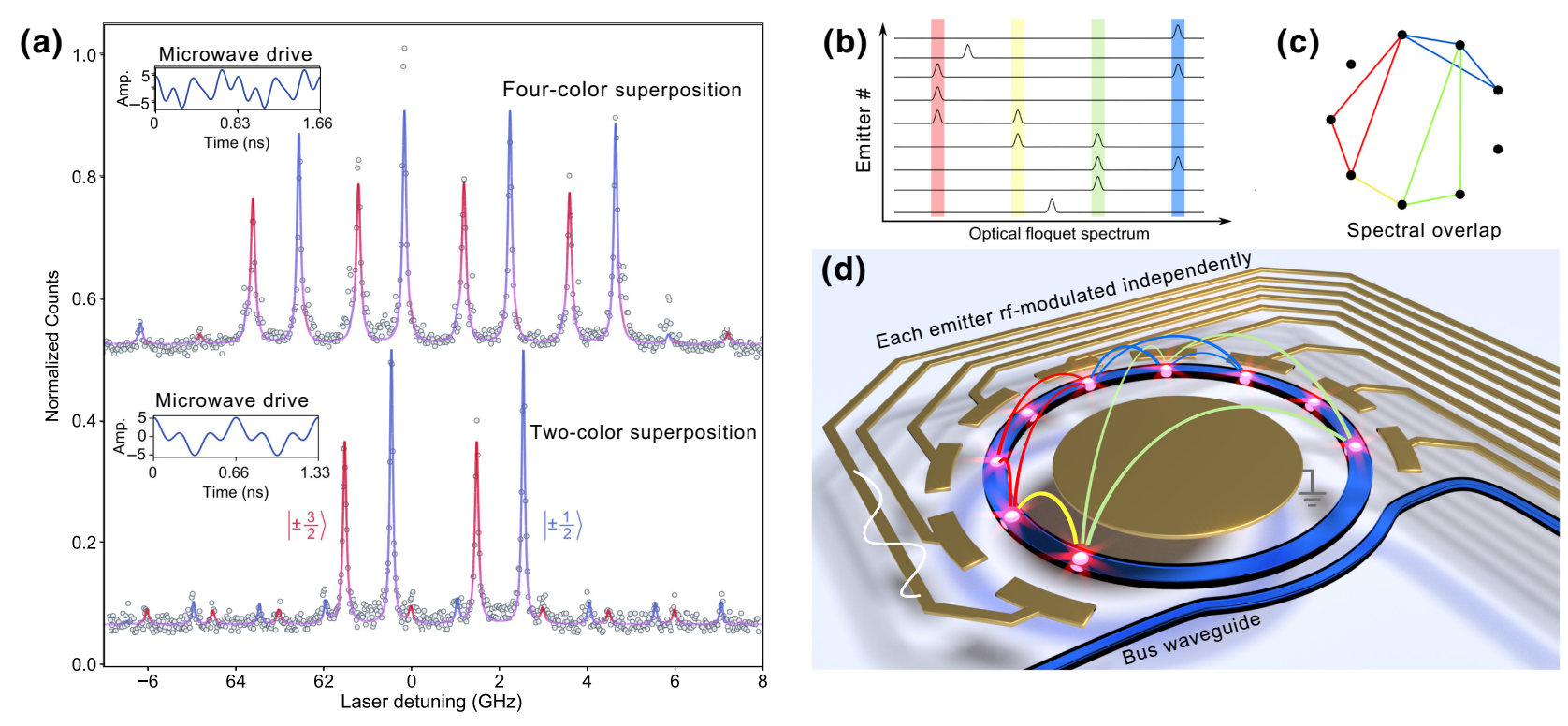

FIG. 6. Quantum information processing with spectrally reconfigurable defects. (a) By rapidly modulating an optical transition, engineering of exotic photon emission spectra from color centers was demonstrated [54]. Via optimized Stark modulation (insets), the $\mathrm{V}_{\mathrm{Si}}$ has been engineered to emit photons in a superposition of 4 (top) and 2 (bottom) colors. (Reproduced from Ref. [54]). Based on this approach one may design nearly arbitrary reconfigurable connectivity via spectral overlap, by independently controlling color centers interacting together via photon-photon interactions through the resonator. This proposal is illustrated in (b),(c) where nine emitters are prepared in Floquet states to create a specific connectivity via spectral overlap. (d) A concept figure of the possible experimental implementation of this approach.

satisfying the extremely low loss requirements of useful quantum photonic computation.

SiC-on-insulator is a promising candidate to realize a fully integrated defect-based quantum photonic processor, using high $Q / V$ photonic crystal cavities, fast cryogenic optical modulators, and integrated detectors. In order to overcome the challenge of nonunity yield, each quantum node may consist of $N$ redundant cavitycoupled spin-defect elements coupled to a bus waveguide. Using this configuration, one can achieve postselection without any additional cavity-waveguide losses, by tuning all but one working node away from the quantum network operation frequency. If necessary, a similar approach can be employed for postselection of detectors (which are to be integrated with low- $Q$ resonators or long waveguides to maximize photon absorption probability). Each node is electrically interfaced to tune the defect optical transition and to coherently manipulate the spin. Fast cryogenic modulators and switches based on a directional coupler or resonator drop-filter configuration can be integrated directly into the $\mathrm{SiC}$ platform, taking advantage of its electro-optic effect. Cryogenic integration based on this approach has only recently been demonstrated [145]. To increase the bandwidth and decrease the footprint, an additional electro-optically active layer, such as barium titanate $\left(\mathrm{BaTiO}_{3}\right)$ [145], can be sputtered and patterned in an adiabatic taper atop the $\mathrm{SiC}$ waveguide to minimize scattering loss. Finally, regardless of the optical frequency of operation of the quantum processor, efficient frequency conversion to the telecommunications band using the strong intrinsic second-order nonlinearity of $\mathrm{SiC}(12 \mathrm{pm} / \mathrm{V})$ [115] would prepare the optically encoded quantum information for long-distance communication.

In the development of scalable quantum photonic circuits, an emerging technique to device engineering - photonics inverse design [146-148] — will likely play an important role. The near-unity fidelities achieved with traditional photonics design, as mentioned above, are limited to simple photonic building blocks such as a directional coupler. However, for more complex tasks, inverse design offers a novel approach for performing targeted optimization against multiple metrics, including robustness to fabrication imperfections (thus improving the yield) and fabrication constraints [149]. For example, a traditional adiabatic taper between photonic layers [142] is extremely sensitive to misalignment, precluding efficient interlayer coupling in pick and place; this limitation is an opportunity to apply inverse design. The powerful functionality of inverse design has been used to demonstrate efficient mode conversion [150] and free-space coupling [151], even under stringent fabrication constraints [152]. The simultaneous inverse design of photonic structures and embedded color centers may enable a fully solid-state 
implementation of a quantum simulator recently proposed for trapped atoms [153].

Finally, we briefly address some new approaches to QIP that may be enabled with recent advancements in colorcenter control and integrated photonics. The recent proposals in quantum simulation based on Floquet state engineering for the superconducting qubit platform $[154,155]$ may find similar implementation in color-center photonics. By coupling an array of emitters to a single nanophotonic resonator, it may be possible to encode arbitrary couplings between them in the frequency basis, taking advantage of nontrivial, multipeaked emission spectra to connect multiple emitters in nontrivial topologies, as shown in Fig. 6. A similar drive-optimization approach may also be used to overcome inhomogeneous broadening in emitter ensembles where individual emitters cannot be controlled but rather a single, global drive signal must be used [54].

\section{CONCLUSION}

In summary, spin-based photonic technologies for quantum computing will likely operate in the network architecture (Fig. 1) and will require the integration of spin-qubit registers with high-quality photonic structures and efficient photon detectors to reduce the total photon loss below the demanding thresholds for quantum computing [15]. While there may be numerous approaches for achieving this goal, we believe that a fully chip-integrated quantum photonic platform holds the most promise, as this approach is most scalable and avoids additional coupling loss from waveguide interconnects. $\mathrm{SiC}$ has emerged as a promising platform for realizing this technology, with demonstrations of wafer-scale integration of high-quality emitters into semiconductor junctions [63], isotopic engineering for nuclear spin registers [88], indistinguishable single-photon emission [52], and a quantum-grade $\mathrm{SiC}$-on-insulator platform for fabrication of photonic devices [32]. However, several key results must be demonstrated to confirm its promise, starting with the integration of large arrays of tunable narrow-linewidth emitters into nanostructures.

\section{ACKNOWLEDGMENTS}

We thank Rahul Trivedi, Shuo Sun, Kiyoul Yang, Sophia Economou, Wenzheng Dong, Florian Kaiser, Oney O. Soykal, Shahriar Aghaeimeibodi, Daniel Riedel, Alexander D. White, Tian Zhong, and Yizhong Huang for insightful discussions. This work is funded in part by the U.S. Department of Energy, Office of Science, under Awards DE-SC0019174 and DE-Ac02-76SF00515; and the National Science Foundation under Grants No. 1839056 and No. EFRI-1741660; D.M.L. acknowledges the Fong Stanford Graduate Fellowship (SGF) and the National Defense Science and Engineering Graduate Fellowship. M.A.G. acknowledges the Albion Hewlett SGF and the NSF Graduate Research Fellowship.
[1] Frank Arute, Kunal Arya, Ryan Babbush, Dave Bacon, Joseph C. Bardin, Rami Barends, Rupak Biswas, Sergio Boixo, Fernando G. S. L. Brandao, and David A. Buell, et al., Quantum supremacy using a programmable superconducting processor, Nature 574, 505 (2019).

[2] Jiehang Zhang, Guido Pagano, Paul W. Hess, Antonis Kyprianidis, Patrick Becker, Harvey Kaplan, Alexey V. Gorshkov, Z.-X. Gong, and Christopher Monroe, Observation of a many-body dynamical phase transition with a 53-qubit quantum simulator, Nature 551, 601 (2017).

[3] Hannes Bernien, Sylvain Schwartz, Alexander Keesling, Harry Levine, Ahmed Omran, Hannes Pichler, Soonwon Choi, Alexander S. Zibrov, Manuel Endres, and Markus Greiner, et al., Probing many-body dynamics on a 51-atom quantum simulator, Nature 551, 579 (2017).

[4] Nicholas C. Harris, Gregory R. Steinbrecher, Mihika Prabhu, Yoav Lahini, Jacob Mower, Darius Bunandar, Changchen Chen, Franco N. C. Wong, Tom Baehr-Jones, and Michael Hochberg, et al., Quantum transport simulations in a programmable nanophotonic processor, Nat. Photonics 11, 447 (2017).

[5] Mete Atatüre, Dirk Englund, Nick Vamivakas, Sang-Yun Lee, and Joerg Wrachtrup, Material platforms for spinbased photonic quantum technologies, Nat. Rev. Mater. 3, 38 (2018).

[6] David D. Awschalom, Ronald Hanson, Jörg Wrachtrup, and Brian B. Zhou, Quantum technologies with optically interfaced solid-state spins, Nat. Photonics 12, 516 (2018).

[7] Jianwei Wang, Fabio Sciarrino, Anthony Laing, and Mark G. Thompson, Integrated photonic quantum technologies, Nat. Photonics 14, 1 (2019).

[8] Ali W Elshaari, Wolfram Pernice, Kartik Srinivasan, Oliver Benson, and Val Zwiller, Hybrid integrated quantum photonic circuits, Nat. Photonics 14, 1 (2020).

[9] Manjin Zhong, Morgan P. Hedges, Rose L. Ahlefeldt, John G. Bartholomew, Sarah E. Beavan, Sven M. Wittig, Jevon J. Longdell, and Matthew J. Sellars, Optically addressable nuclear spins in a solid with a six-hour coherence time, Nature 517, 177 (2015).

[10] C. E. Bradley, J. Randall, M. H. Abobeih, R. C. Berrevoets, M. J. Degen, M. A. Bakker, M. Markham, D. J. Twitchen, and T. H. Taminiau, A Ten-Qubit Solid-State Spin Register with Quantum Memory up to one Minute, Phys. Rev. X 9, 031045 (2019).

[11] Kevin C. Miao, Joseph P. Blanton, Christopher P. Anderson, Alexandre Bourassa, Alexander L. Crook, Gary Wolfowicz, Hiroshi Abe, Takeshi Ohshima, and David D. Awschalom, Universal coherence protection in a solidstate spin qubit, Science 369, 1493 (2020).

[12] Bas Hensen, Hannes Bernien, Anaïs E. Dréau, Andreas Reiserer, Norbert Kalb, Machiel S. Blok, Just Ruitenberg, Raymond F. L. Vermeulen, Raymond N. Schouten, and Carlos Abellán, et al., Loophole-free bell inequality violation using electron spins separated by 1.3 kilometres, Nature 526, 682 (2015).

[13] Kae Nemoto, Michael Trupke, Simon J. Devitt, Ashley M. Stephens, Burkhard Scharfenberger, Kathrin Buczak, Tobias Nöbauer, Mark S. Everitt, Jörg Schmiedmayer, and William J. Munro, Photonic Architecture for Scalable Quantum Information Processing in Diamond, Phys. Rev. X 4, 031022 (2014). 
[14] Naomi H. Nickerson, Ying Li, and Simon C. Benjamin, Topological quantum computing with a very noisy network and local error rates approaching one percent, Nat. Commun. 4, 1 (2013).

[15] Naomi H. Nickerson, Joseph F. Fitzsimons, and Simon C. Benjamin, Freely Scalable Quantum Technologies Using Cells of 5-To-50 Qubits with Very Lossy and Noisy Photonic Links, Phys. Rev. X 4, 041041 (2014).

[16] Donovan Buterakos, Edwin Barnes, and Sophia E. Economou, Deterministic Generation of All-Photonic Quantum Repeaters from Solid-State Emitters, Phys. Rev. X 7, 041023 (2017).

[17] Antonio Russo, Edwin Barnes, and Sophia E. Economou, Photonic graph state generation from quantum dots and color centers for quantum communications, Phys. Rev. B 98, 085303 (2018).

[18] Ido Schwartz, Dan Cogan, Emma R. Schmidgall, Yaroslav Don, Liron Gantz, Oded Kenneth, Netanel H. Lindner, and David Gershoni, Deterministic generation of a cluster state of entangled photons, Science 354, 434 (2016).

[19] Sreraman Muralidharan, Linshu Li, Jungsang Kim, Norbert Lütkenhaus, Mikhail D. Lukin, and Liang Jiang, Optimal architectures for long distance quantum communication, Sci. Rep. 6, 20463 (2016).

[20] Johannes Borregaard, Hannes Pichler, Tim Schröder, Mikhail D. Lukin, Peter Lodahl, and Anders S. Sørensen, One-Way Quantum Repeater Based on NearDeterministic Photon-Emitter Interfaces, Phys. Rev. X 10, 021071 (2020).

[21] Mihir K. Bhaskar, Ralf Riedinger, Bartholomeus Machielse, David S. Levonian, Christian T. Nguyen, Erik N. Knall, Hongkun Park, Dirk Englund, Marko Lončar, and Denis D. Sukachev, et al., Experimental demonstration of memory-enhanced quantum communication, Nature 580, 60 (2020).

[22] Tian Zhong, Jonathan M. Kindem, John G. Bartholomew, Jake Rochman, Ioana Craiciu, Evan Miyazono, Marco Bettinelli, Enrico Cavalli, Varun Verma, and Sae Woo Nam, et al., Nanophotonic rare-earth quantum memory with optically controlled retrieval, Science 357, 1392 (2017).

[23] Norbert Kalb, Andreas A. Reiserer, Peter C. Humphreys, Jacob J. W. Bakermans, Sten J. Kamerling, Naomi H. Nickerson, Simon C. Benjamin, Daniel J. Twitchen, Matthew Markham, and Ronald Hanson, Entanglement distillation between solid-state quantum network nodes, Science 356, 928 (2017).

[24] Ruffin E. Evans, Mihir K. Bhaskar, Denis D. Sukachev, Christian T. Nguyen, Alp Sipahigil, Michael J. Burek, Bartholomeus Machielse, Grace H. Zhang, Alexander S. Zibrov, and Edward Bielejec, et al., Photon-mediated interactions between quantum emitters in a diamond nanocavity, Science 362, 662 (2018).

[25] Jonathan M. Kindem, Andrei Ruskuc, John G. Bartholomew, Jake Rochman, Yan Qi Huan, and Andrei Faraon, Control and single-shot readout of an ion embedded in a nanophotonic cavity, Nature 580, 201 (2020).

[26] Mouktik Raha, Songtao Chen, Christopher M. Phenicie, Salim Ourari, Alan M. Dibos, and Jeff D. Thompson,
Optical quantum nondemolition measurement of a single rare earth ion qubit, Nat. Commun. 11, 1 (2020).

[27] Cheng Wang, Mian Zhang, Xi Chen, Maxime Bertrand, Amirhassan Shams-Ansari, Sethumadhavan Chandrasekhar, Peter Winzer, and Marko Lončar, Integrated lithium niobate electro-optic modulators operating at cmos-compatible voltages, Nature 562, 101 (2018).

[28] Martijn J. R. Heck, Jared F. Bauters, Michael L. Davenport, Daryl T. Spencer, and John E. Bowers, Ultra-low loss waveguide platform and its integration with silicon photonics, Laser Photon. Rev. 8, 667 (2014).

[29] Junqiu Liu, Hao Tian, Erwan Lucas, Arslan S. Raja, Grigory Lihachev, Rui Ning Wang, Jijun He, Tianyi Liu, Miles H. Anderson, and Wenle Weng, et al., Monolithic piezoelectric control of soliton microcombs, Nature 583, 385 (2020).

[30] Junqiu Liu, Guanhao Huang, Rui Ning Wang, Jijun He, Arslan S. Raja, Tianyi Liu, Nils J. Engelsen, and Tobias J. Kippenberg, High-yield wafer-scale fabrication of ultralow-loss, dispersion-engineered silicon nitride photonic circuits, arXiv preprint arXiv:2005.13949 (2020).

[31] Stefania Castelletto and Alberto Boretti, Silicon carbide color centers for quantum applications, J. Phys.: Photonics 2, 022001 (2020).

[32] Daniil M. Lukin, Constantin Dory, Melissa A. Guidry, Ki Youl Yang, Sattwik Deb Mishra, Rahul Trivedi, Marina Radulaski, Shuo Sun, Dries Vercruysse, and Geun Ho Ahn, et al., 4h-silicon-carbide-on-insulator for integrated quantum and nonlinear photonics, Nat. Photonics 14, 330 (2020).

[33] Bong-Shik Song, Takashi Asano, Seungwoo Jeon, Heungjoon Kim, Changxuan Chen, Dongyeon Daniel Kang, and Susumu Noda, Ultrahigh-q photonic crystal nanocavities based on 4h silicon carbide, Optica 6, 991 (2019).

[34] Melissa A. Guidry, Ki Youl Yang, Daniil M. Lukin, Ashot Markosyan, Joshua Yang, Martin M. Fejer, and Jelena Vučković, Optical parametric oscillation in silicon carbide nanophotonics, Optica 7, 1139 (2020).

[35] Roland Nagy, Matthias Niethammer, Matthias Widmann, Yu-Chen Chen, Péter Udvarhelyi, Cristian Bonato, Jawad Ul Hassan, Robin Karhu, Ivan G. Ivanov, and Nguyen Tien Son, et al., High-fidelity spin and optical control of single silicon-vacancy centres in silicon carbide, Nat. Commun. 10, 1 (2019).

[36] Rui Vasconcelos, Sarah Reisenbauer, Cameron Salter, Georg Wachter, Daniel Wirtitsch, Jörg Schmiedmayer, Philip Walther, and Michael Trupke, Scalable spin-photon entanglement by time-to-polarization conversion, npj Quantum Inf. 6, 1 (2020).

[37] Nguyen T. Son, Christopher P. Anderson, Alexandre Bourassa, Kevin C. Miao, Charles Babin, Matthias Widmann, Matthias Niethammer, Jawad Ul Hassan, Naoya Morioka, and Ivan G. Ivanov, et al., Developing silicon carbide for quantum spintronics, Appl. Phys. Lett. 116, 190501 (2020).

[38] Iman Esmaeil Zadeh, Johannes W. N. Los, Ronan B. M. Gourgues, Jin Chang, Ali W. Elshaari, Julien Romain Zichi, Yuri J. van Staaden, Jereon P. E. Swens, Nima Kalhor, and Antonio Guardiani, et al., Efficient 
single-photon detection with 7.7 ps time resolution for photon correlation measurements, ACS Photonics 7, 1780 (2020).

[39] J. D. Joannopoulos, S. G.Johnson, J. N. Winn and R. D. Meade, Photonic crystals: Molding the flow of light (Princeton University Press, Princeton, 1995), 2nd ed.

[40] Marta Arcari, Immo Söllner, Alisa Javadi, S. Lindskov Hansen, Sahand Mahmoodian, Jin Liu, Henri Thyrrestrup, Eun Hye Lee, Jin Dong Song, and Søren Stobbe, et al., Near-Unity Coupling Efficiency of a Quantum Emitter to a Photonic Crystal Waveguide, Phys. Rev. Lett. 113, 093603 (2014).

[41] Edo Waks and Jelena Vuckovic, Dipole Induced Transparency in Drop-Filter Cavity-Waveguide Systems, Phys. Rev. Lett. 96, 153601 (2006).

[42] Wenzheng Dong, M. W. Doherty, and Sophia E. Economou, Spin polarization through intersystem crossing in the silicon vacancy of silicon carbide, Phys. Rev. B 99, 184102 (2019).

[43] P. Neumann, R. Kolesov, B. Naydenov, J. Beck, F. Rempp, M. Steiner, V. Jacques, G. Balasubramanian, M. L. Markham, and D. J. Twitchen, et al., Quantum register based on coupled electron spins in a room-temperature solid, Nat. Phys. 6, 249 (2010).

[44] E. Sörman, N. T. Son, W. M. Chen, O. Kordina, Christer Hallin, and Erik Janzén, Silicon vacancy related defect in 4h and 6h sic, Phys. Rev. B 61, 2613 (2000).

[45] Pavel G. Baranov, Anna P. Bundakova, Alexandra A. Soltamova, Sergei B. Orlinskii, Igor V. Borovykh, Rob Zondervan, Rogier Verberk, and Jan Schmidt, Silicon vacancy in sic as a promising quantum system for singledefect and single-photon spectroscopy, Phys. Rev. B 83, 125203 (2011).

[46] V. A. Soltamov, B. V. Yavkin, D. O. Tolmachev, R. A. Babunts, A. G. Badalyan, V. Yu Davydov, E. N. Mokhov, I. I. Proskuryakov, S. B. Orlinskii, and P. G. Baranov, Optically Addressable Silicon Vacancy-Related Spin Centers in Rhombic Silicon Carbide with High Breakdown Characteristics and Endor Evidence of Their Structure, Phys. Rev. Lett. 115, 247602 (2015).

[47] D. Simin, H. Kraus, A. Sperlich, T. Ohshima, G. V. Astakhov, and V. Dyakonov, Locking of electron spin coherence above $20 \mathrm{~ms}$ in natural silicon carbide, Phys. Rev. B 95, 161201 (2017).

[48] Hunter B. Banks, Öney O. Soykal, Rachael L. MyersWard, D. Kurt Gaskill, T. L. Reinecke, and Samuel G. Carter, Resonant Optical Spin Initialization and Readout of Single Silicon Vacancies in 4 H-si c, Phys. Rev. Appl. 11, 024013 (2019).

[49] Joel Davidsson, Viktor Ivády, Rickard Armiento, Takeshi Ohshima, N. T. Son, Adam Gali, and Igor A. Abrikosov, Identification of divacancy and silicon vacancy qubits in 6h-sic, Appl. Phys. Lett. 114, 112107 (2019).

[50] Péter Udvarhelyi, Gergö Thiering, Naoya Morioka, Charles Babin, Florian Kaiser, Daniil Lukin, Takeshi Ohshima, Jawad Ul-Hassan, Nguyen Tien Son, and Jelena Vučković, et al., Vibronic States and Their Effect on the Temperature and Strain Dependence of SiliconVacancy Qubits in 4 H-si c, Phys. Rev. Appl. 13, 054017 (2020).
[51] Z. Shang, A. Hashemi, Y. Berencén, H.-P. Komsa, P. Erhart, S. Zhou, M. Helm, A. V. Krasheninnikov, and G. V. Astakhov, Local vibrational modes of si vacancy spin qubits in sic, Phys. Rev. B 101, 144109 (2020).

[52] Naoya Morioka, Charles Babin, Roland Nagy, Izel Gediz, Erik Hesselmeier, Di Liu, Matthew Joliffe, Matthias Niethammer, Durga Dasari, and Vadim Vorobyov, et al., Spin-controlled generation of indistinguishable and distinguishable photons from silicon vacancy centres in silicon carbide, Nat. Commun. 11, 1 (2020).

[53] Maximilian Rühl, Lena Bergmann, Michael Krieger, and Heiko B. Weber, Stark tuning of the silicon vacancy in silicon carbide, Nano Lett. 20, 658 (2019).

[54] Daniil M. Lukin, Alexander D. White, Melissa A. Guidry, Rahul Trivedi, Naoya Morioka, Charles Babin, Jawad Ul Hassan, Nguyen Tien Son, Takeshi Ohshima, and Praful K. Vasireddy, et al., Spectrally reconfigurable quantum emitters enabled by optimized fast modulation, npj Quantum Inf. 6, 80 (2020).

[55] William F. Koehl, Bob B. Buckley, F. Joseph Heremans, Greg Calusine, and David D. Awschalom, Room temperature coherent control of defect spin qubits in silicon carbide, Nature 479, 84 (2011).

[56] Abram L. Falk, Bob B. Buckley, Greg Calusine, William F. Koehl, Viatcheslav V. Dobrovitski, Alberto Politi, Christian A. Zorman, Philip X.-L. Feng, and David D. Awschalom, Polytype control of spin qubits in silicon carbide, Nat. Commun. 4, 1 (2013).

[57] David J. Christle, Abram L. Falk, Paolo Andrich, Paul V. Klimov, Jawad Ul Hassan, Nguyen T. Son, Erik Janzén, Takeshi Ohshima, and David D. Awschalom, Isolated electron spins in silicon carbide with millisecond coherence times, Nat. Mater. 14, 160 (2015).

[58] Abram L. Falk, Paul V. Klimov, Viktor Ivády, Krisztián Szász, David J. Christle, William F. Koehl, Ádám Gali, and David D. Awschalom, Optical Polarization of Nuclear Spins in Silicon Carbide, Phys. Rev. Lett. 114, 247603 (2015).

[59] Paul V. Klimov, Abram L. Falk, David J. Christle, Viatcheslav V. Dobrovitski, and David D. Awschalom, Quantum entanglement at ambient conditions in a macroscopic solid-state spin ensemble, Sci. Adv. 1, e1501015 (2015).

[60] Viktor Ivády, Paul V. Klimov, Kevin C. Miao, Abram L. Falk, David J. Christle, Krisztián Szász, Igor A. Abrikosov, David D. Awschalom, and Adam Gali, HighFidelity Bidirectional Nuclear Qubit Initialization in sic, Phys. Rev. Lett. 117, 220503 (2016).

[61] David J. Christle, Paul V. Klimov, F. Charles, Krisztián Szász, Viktor Ivády, Valdas Jokubavicius, Jawad U1 Hassan, Mikael Syväjärvi, William F. Koehl, and Takeshi Ohshima, et al., Isolated Spin Qubits in sic with a HighFidelity Infrared Spin-To-Photon Interface, Phys. Rev. X 7, 021046 (2017).

[62] Kevin C. Miao, Alexandre Bourassa, Christopher P. Anderson, Samuel J. Whiteley, Alexander L. Crook, Sam L. Bayliss, Gary Wolfowicz, Gergö Thiering, Péter Udvarhelyi, and Viktor Ivády, et al., Electrically driven optical interferometry with spins in silicon carbide, Sci. Adv. 5, eaay0527 (2019). 
[63] Christopher P. Anderson, Alexandre Bourassa, Kevin C. Miao, Gary Wolfowicz, Peter J. Mintun, Alexander L. Crook, Hiroshi Abe, Jawad Ul Hassan, Nguyen T. Son, and Takeshi Ohshima, et al., Electrical and optical control of single spins integrated in scalable semiconductor devices, Science 366, 1225 (2019).

[64] H. J. Von Bardeleben, J. L. Cantin, A. Csóré, A. Gali, E. Rauls, and U. Gerstmann, Nv centers in $3 \mathrm{c}, 4 \mathrm{~h}$, and $6 \mathrm{~h}$ silicon carbide: A variable platform for solid-state qubits and nanosensors, Phys. Rev. B 94, 121202 (2016).

[65] Soroush Abbasi Zargaleh, Sophie Hameau, Benoit Eble, F. Margaillan, Hans Jürgen von Bardeleben, Jean-Louis Cantin, and Weibo Gao, Nitrogen vacancy center in cubic silicon carbide: A promising qubit in the $1.5 \mu \mathrm{m}$ spectral range for photonic quantum networks, Phys. Rev. B 98, 165203 (2018).

[66] S. A. Zargaleh, B. Eble, S. Hameau, J.-L. Cantin, L. Legrand, M. Bernard, F. Margaillan, J.-S. Lauret, J.-F. Roch, and H. J. Von Bardeleben, et al., Evidence for nearinfrared photoluminescence of nitrogen vacancy centers in 4 h-sic, Phys. Rev. B 94, 060102 (2016).

[67] A Csóré, H. J. Von Bardeleben, J. L. Cantin, and A. Gali, Characterization and formation of nv centers in $3 \mathrm{c}, 4 \mathrm{~h}$, and 6 h sic: An ab initio study, Phys. Rev. B 96, 085204 (2017).

[68] Jun-Feng Wang, Fei-Fei Yan, Qiang Li, Zheng-Hao Liu, He Liu, Guo-Ping Guo, Li-Ping Guo, Xiong Zhou, JinMing Cui, and Jian Wang, et al., Coherent Control of Nitrogen-Vacancy Center Spins in Silicon Carbide at Room Temperature, Phys. Rev. Lett. 124, 223601 (2020).

[69] William F. Koehl, Berk Diler, Samuel J. Whiteley, Alexandre Bourassa, N. Tien Son, Erik Janzén, and David D. Awschalom, Resonant optical spectroscopy and coherent control of cr 4+ spin ensembles in sic and gan, Phys. Rev. B 95, 035207 (2017).

[70] Berk Diler, Samuel J. Whiteley, Christopher P. Anderson, Gary Wolfowicz, Marie E. Wesson, Edward S. Bielejec, F. Joseph Heremans, and David D. Awschalom, Coherent control and high-fidelity readout of chromium ions in commercial silicon carbide, npj Quantum Inf. 6, 1 (2020).

[71] Lukas Spindlberger, András Csóré, Gergö Thiering, S. Putz, Robin Karhu, J. Ul Hassan, N. T. Son, T. Fromherz, A. Gali, and M. Trupke, Optical Properties of Vanadium in 4h Silicon Carbide for Quantum Technology, Phys. Rev. Appl. 12, 014015 (2019).

[72] Gary Wolfowicz, Christopher P. Anderson, Berk Diler, Oleg G. Poluektov, F. Joseph Heremans, and David D. Awschalom, Vanadium spin qubits as telecom quantum emitters in silicon carbide, arXiv:1908.09817 (2019).

[73] H. Kraus, D. Simin, C. Kasper, Y. Suda, S. Kawabata, W. Kada, T. Honda, Y. Hijikata, T. Ohshima, and V. Dyakonov, et al., Three-dimensional proton beam writing of optically active coherent vacancy spins in silicon carbide, Nano Lett. 17, 2865 (2017).

[74] Yu-Chen Chen, Patrick S. Salter, Matthias Niethammer, Matthias Widmann, Florian Kaiser, Roland Nagy, Naoya Morioka, Charles Babin, Jürgen Erlekampf, and Patrick Berwian, et al., Laser writing of scalable single color centers in silicon carbide, Nano Lett. 19, 2377 (2019).
[75] H. Kraus, V. A. Soltamov, D. Riedel, S. Väth, F. Fuchs, A. Sperlich, P. G. Baranov, V. Dyakonov, and G. V. Astakhov, Room-temperature quantum microwave emitters based on spin defects in silicon carbide, Nat. Phys. 10, 157 (2014).

[76] D. Simin, F. Fuchs, H. Kraus, A. Sperlich, P. G. Baranov, G. V. Astakhov, and V. Dyakonov, High-Precision AngleResolved Magnetometry with Uniaxial Quantum Centers in Silicon Carbide, Phys. Rev. Appl. 4, 014009 (2015).

[77] D. Simin, V. A. Soltamov, A. V. Poshakinskiy, A. N. Anisimov, R. A. Babunts, D. O. Tolmachev, E. N. Mokhov, M. Trupke, S. A. Tarasenko, and A. Sperlich, et al., All-Optical dc Nanotesla Magnetometry Using Silicon Vacancy Fine Structure in Isotopically Purified Silicon Carbide, Phys. Rev. X 6, 031014 (2016).

[78] V. A. Soltamov, C. Kasper, A. V. Poshakinskiy, A. N. Anisimov, E. N. Mokhov, A. Sperlich, S. A. Tarasenko, P. G. Baranov, G. V. Astakhov, and V. Dyakonov, Excitation and coherent control of spin qudit modes in silicon carbide at room temperature, Nat. Commun. 10, 1 (2019).

[79] Matthias Widmann, Sang-Yun Lee, Torsten Rendler, Nguyen Tien Son, Helmut Fedder, Seoyoung Paik, LiPing Yang, Nan Zhao, Sen Yang, and Ian Booker, et al., Coherent control of single spins in silicon carbide at room temperature, Nat. Mater. 14, 164 (2015).

[80] F. Fuchs, B. Stender, M. Trupke, D. Simin, J. Pflaum, V. Dyakonov, and G. V. Astakhov, Engineering nearinfrared single-photon emitters with optically active spins in ultrapure silicon carbide, Nat. Commun. 6, 1 (2015).

[81] C. Kasper, D. Klenkert, Z. Shang, D. Simin, A. Gottscholl, A. Sperlich, H. Kraus, C. Schneider, S. Zhou, and M. Trupke, et al., Influence of Irradiation on Defect Spin Coherence in Silicon Carbide, Phys. Rev. Appl. 13, 044054 (2020).

[82] Junfeng Wang, Yu Zhou, Xiaoming Zhang, Fucai Liu, Yan Li, Ke Li, Zheng Liu, Guanzhong Wang, and Weibo Gao, Efficient Generation of an Array of Single SiliconVacancy Defects in Silicon Carbide, Phys. Rev. Appl. 7, 064021 (2017).

[83] ÖO Soykal, Pratibha Dev, and Sophia E. Economou, Silicon vacancy center in $4 \mathrm{~h}$-sic: Electronic structure and spin-photon interfaces, Phys. Rev. B 93, 081207 (2016).

[84] Sophia E. Economou and Pratibha Dev, Spin-photon entanglement interfaces in silicon carbide defect centers, Nanotechnology 27, 504001 (2016).

[85] Spectrally Stable Defect Qubits with no Inversion Symmetry for Robust Spin-To-Photon Interface, Phys. Rev. Appl. 11, 044022 (2019).

[86] Matthias Niethammer, Matthias Widmann, Torsten Rendler, Naoya Morioka, Yu-Chen Chen, Rainer Stöhr, Jawad Ul Hassan, Shinobu Onoda, Takeshi Ohshima, and Sang-Yun Lee, et al., Coherent electrical readout of defect spins in silicon carbide by photo-ionization at ambient conditions, Nat. Commun. 10, 1 (2019).

[87] Matthias Widmann, Matthias Niethammer, Dmitry $\mathrm{Yu}$ Fedyanin, Igor A. Khramtsov, Torsten Rendler, Ian D. Booker, Jawad Ul Hassan, Naoya Morioka, Yu-Chen Chen, and Ivan G. Ivanov, et al., Electrical charge state manipulation of single silicon vacancies in a silicon carbide quantum optoelectronic device, Nano Lett. 19, 7173 (2019). 
[88] Alexandre Bourassa, Christopher P. Anderson, Kevin C. Miao, Mykyta Onizhuk, He Ma, Alexander L. Crook, Hiroshi Abe, Jawad Ul-Hassan, Takeshi Ohshima, and Nguyen T. Son, et al., Entanglement and control of single nuclear spins in isotopically engineered silicon carbide, Nat. Mater. 19, 1 (2020).

[89] P. V. Klimov, A. L. Falk, B. B. Buckley, and D. D. Awschalom, Electrically Driven Spin Resonance in Silicon Carbide Color Centers, Phys. Rev. Lett. 112, 087601 (2014).

[90] Abram L. Falk, Paul V. Klimov, Bob B. Buckley, Viktor Ivády, Igor A. Abrikosov, Greg Calusine, William F. Koehl, Ádám Gali, and David D. Awschalom, Electrically and Mechanically Tunable Electron Spins in Silicon Carbide Color Centers, Phys. Rev. Lett. 112, 187601 (2014).

[91] Samuel J. Whiteley, Gary Wolfowicz, Christopher P. Anderson, Alexandre Bourassa, He Ma, Meng Ye, Gerwin Koolstra, Kevin J. Satzinger, Martin V. Holt, and F. Joseph Heremans, et al., Spin-phonon interactions in silicon carbide addressed by gaussian acoustics, Nat. Phys. 15, 490 (2019).

[92] L. Di Cioccio, F. Letertre, Y. Le Tiec, A. M. Papon, C. Jaussaud, and M. Bruel, Silicon carbide on insulator formation by the smart-cut process, Mater. Sci. Eng.: B 46, 349 (1997).

[93] Bong-Shik Song, Shota Yamada, Takashi Asano, and Susumu Noda, Demonstration of two-dimensional photonic crystals based on silicon carbide, Opt. Express 19, 11084 (2011).

[94] Shota Yamada, Bong-Shik Song, Seungwoo Jeon, Jeremy Upham, Yoshinori Tanaka, Takashi Asano, and Susumu Noda, Second-harmonic generation in a silicon-carbidebased photonic crystal nanocavity, Opt. Lett. 39, 1768 (2014).

[95] Jaime Cardenas, Mengjie Yu, Yoshitomo Okawachi, Carl B. Poitras, Ryan K. W. Lau, Avik Dutt, Alexander L. Gaeta, and Michal Lipson, Optical nonlinearities in highconfinement silicon carbide waveguides, Opt. Lett. 40, 4138 (2015).

[96] Ailun Yi, Yi Zheng, Hao Huang, Jiajie Lin, Youquan Yan, Tiangui You, Kai Huang, Shibin Zhang, Chen Shen, and Min Zhou, et al., Wafer-scale 4h-silicon carbideon-insulator (4h-sicoi) platform for nonlinear integrated optical devices, Opt. Mater. (Amst) 107, 109990 (2020).

[97] Yi Zheng, Minhao Pu, Ailun Yi, Bingdong Chang, Tiangui You, Kai Huang, Ayman N. Kamel, Martin R. Henriksen, Asbjørn A. Jørgensen, and Xin Ou, et al., Highquality factor, high-confinement microring resonators in 4h-silicon carbide-on-insulator, Opt. Express 27, 13053 (2019).

[98] Jaime Cardenas, Mian Zhang, Christopher T. Phare, Shreyas Y. Shah, Carl B. Poitras, Biswajeet Guha, and Michal Lipson, High q sic microresonators, Opt. Express 21, 16882 (2013).

[99] Marina Radulaski, Thomas M. Babinec, Sonia Buckley, Armand Rundquist, J. Provine, Kassem Alassaad, Gabriel Ferro, and Jelena Vučković, Photonic crystal cavities in cubic (3c) polytype silicon carbide films, Opt. Express 21, 32623 (2013).
[100] Xiyuan Lu, Jonathan Y. Lee, Philip X.-L. Feng, and Qiang Lin, High q silicon carbide microdisk resonator, Appl. Phys. Lett. 104, 181103 (2014).

[101] Tianren Fan, Hesam Moradinejad, Xi Wu, Ali A. Eftekhar, and Ali Adibi, High-q integrated photonic microresonators on 3c-sic-on-insulator (sicoi) platform, Opt. Express 26, 25814 (2018).

[102] Tianren Fan, Xi Wu, Ali A. Eftekhar, Matteo Bosi, Hesam Moradinejad, Eric V. Woods, and Ali Adibi, High-quality integrated microdisk resonators in the visible-to-nearinfrared wavelength range on a $3 \mathrm{c}$-silicon carbide-oninsulator platform, Opt. Lett. 45, 153 (2020).

[103] Greg Calusine, Alberto Politi, and David D. Awschalom, Cavity-Enhanced Measurements of Defect Spins in Silicon Carbide, Phys. Rev. Appl. 6, 014019 (2016).

[104] Michael J. Burek, Yiwen Chu, Madelaine S. Z. Liddy, Parth Patel, Jake Rochman, Srujan Meesala, Wooyoung Hong, Qimin Quan, Mikhail D. Lukin, and Marko Lončar, High quality-factor optical nanocavities in bulk singlecrystal diamond, Nat. Commun. 5, 1 (2014).

[105] Bong-Shik Song, Seungwoo Jeon, Heungjoon Kim, Dongyeon Daniel Kang, Takashi Asano, and Susumu Noda, High-q-factor nanobeam photonic crystal cavities in bulk silicon carbide, Appl. Phys. Lett. 113, 231106 (2018).

[106] David O. Bracher, Xingyu Zhang, and Evelyn L. Hu, Selective purcell enhancement of two closely linked zerophonon transitions of a silicon carbide color center, Proc. Natl. Acad. Sci. 114, 4060 (2017).

[107] Alexander L. Crook, Christopher P. Anderson, Kevin C. Miao, Alexandre Bourassa, Hope Lee, Sam L. Bayliss, David O. Bracher, Xingyu Zhang, Hiroshi Abe, and Takeshi Ohshima, et al., Purcell enhancement of a single silicon carbide color center with coherent spin control, Nano Lett. 20, 3427 (2020).

[108] Lucio Robledo, Lilian Childress, Hannes Bernien, Bas Hensen, Paul F. A. Alkemade, and Ronald Hanson, Highfidelity projective read-out of a solid-state spin quantum register, Nature 477, 574 (2011).

[109] Denis D. Sukachev, Alp Sipahigil, Christian T. Nguyen, Mihir K. Bhaskar, Ruffin E. Evans, Fedor Jelezko, and Mikhail D. Lukin, Silicon-Vacancy Spin Qubit in Diamond: A Quantum Memory Exceeding $10 \mathrm{~ms}$ with Single-Shot State Readout, Phys. Rev. Lett. 119, 223602 (2017).

[110] Michael J. Burek, Charles Meuwly, Ruffin E. Evans, Mihir K. Bhaskar, Alp Sipahigil, Srujan Meesala, Bartholomeus Machielse, Denis D. Sukachev, Christian T. Nguyen, and Jose L. Pacheco, et al., Fiber-Coupled Diamond Quantum Nanophotonic Interface, Phys. Rev. Appl. 8, 024026 (2017).

[111] Bartholomeus Machielse, Stefan Bogdanovic, Srujan Meesala, Scarlett Gauthier, Michael J. Burek, Graham Joe, Michelle Chalupnik, Young-Ik Sohn, Jeffrey Holzgrafe, and Ruffin E. Evans, et al., Quantum Interference of Electromechanically Stabilized Emitters in Nanophotonic Devices, Phys. Rev. X 9, 031022 (2019).

[112] Noel H. Wan, Tsung-Ju Lu, Kevin C. Chen, Michael P. Walsh, Matthew E. Trusheim, Lorenzo De Santis, Eric A. Bersin, Isaac B. Harris, Sara L. Mouradian, and Ian R. 
Christen, et al., Large-scale integration of artificial atoms in hybrid photonic circuits, Nature 583, 226 (2020).

[113] Alison E. Rugar, Constantin Dory, Shahriar Aghaeimeibodi, Haiyu Lu, Shuo Sun, Sattwik Deb Mishra, Zhi-Xun Shen, Nicholas A. Melosh, and Jelena Vučković, Narrowlinewidth tin-vacancy centers in a diamond waveguide, ACS Photonics 7, 2356 (2020).

[114] B. J. M. Hausmann, I. Bulu, V. Venkataraman, P. Deotare, and Marko Lončar, Diamond nonlinear photonics, Nat. Photonics 8, 369 (2014).

[115] Hiroaki Sato, Makoto Abe, Ichiro Shoji, Jun Suda, and Takashi Kondo, Accurate measurements of second-order nonlinear optical coefficients of $6 \mathrm{~h}$ and $4 \mathrm{~h}$ silicon carbide, JOSA B 26, 1892 (2009).

[116] Takashi Asano, Yoshiaki Ochi, Yasushi Takahashi, Katsuhiro Kishimoto, and Susumu Noda, Photonic crystal nanocavity with a q factor exceeding eleven million, Opt. Express 25, 1769 (2017).

[117] Hansuek Lee, Tong Chen, Jiang Li, Ki Youl Yang, Seokmin Jeon, Oskar Painter, and Kerry J. Vahala, Chemically etched ultrahigh-q wedge-resonator on a silicon chip, Nat. Photonics 6, 369 (2012).

[118] Ki Youl Yang, Dong Yoon Oh, Seung Hoon Lee, QiFan Yang, Xu Yi, Boqiang Shen, Heming Wang, and Kerry Vahala, Bridging ultrahigh-q devices and photonic circuits, Nat. Photonics 12, 297 (2018).

[119] Mingxiao Li, Hanxiao Liang, Rui Luo, Yang He, Jingwei Ling, and Qiang Lin, Photon-level tuning of photonic nanocavities, Optica 6, 860 (2019).

[120] Gregory S. MacCabe, Hengjiang Ren, Jie Luo, Justin D. Cohen, Hengyun Zhou, Alp Sipahigil, Mohammad Mirhosseini, and Oskar Painter, Phononic bandgap nano-acoustic cavity with ultralong phonon lifetime, arXiv:1901.04129 (2019).

[121] Kazuhiro Kuruma, Yasutomo Ota, Masahiro Kakuda, Satoshi Iwamoto, and Yasuhiko Arakawa, Surfacepassivated high-q gaas photonic crystal nanocavity with quantum dots, APL Photonics 5, 046106 (2020).

[122] Neeraj Mishra, John Boeckl, Nunzio Motta, and Francesca Iacopi, Graphene growth on silicon carbide: A review, Phys. Status Solidi (A) 213, 2277 (2016).

[123] Bryan Ellis, Marie A. Mayer, Gary Shambat, Tomas Sarmiento, James Harris, Eugene E. Haller, and Jelena Vučković, Ultralow-threshold electrically pumped quantum-dot photonic-crystal nanocavity laser, Nat. Photonics 5, 297 (2011).

[124] Mulpuri V. Rao, J. Tucker, O. W. Holland, N. Papanicolaou, P. H. Chi, J. W. Kretchmer, and M. Ghezzo, Donor ion-implantation doping into sic, J. Electron. Mater. 28, 334 (1999).

[125] H. F. Fotso, A. E. Feiguin, D. D. Awschalom, and V. V. Dobrovitski, Suppressing Spectral Diffusion of Emitted Photons with Optical Pulses, Phys. Rev. Lett. 116, 033603 (2016).

[126] Dolev Bluvstein, Zhiran Zhang, Claire A. McLellan, Nicolas R. Williams, and Ania C. Bleszynski Jayich, Extending the Quantum Coherence of a NearSurface Qubit by Coherently Driving the Paramagnetic Surface Environment, Phys. Rev. Lett. 123, 146804 (2019).
[127] Toan Trong Tran, Carlo Bradac, Alexander S. Solntsev, Milos Toth, and Igor Aharonovich, Suppression of spectral diffusion by anti-Stokes excitation of quantum emitters in hexagonal boron nitride, Appl. Phys. Lett. 115, 071102 (2019).

[128] Xiaodong Yang, Charlton J. Chen, Chad A. Husko, and Chee Wei Wong, Digital resonance tuning of high-q/ v $\mathrm{m}$ silicon photonic crystal nanocavities by atomic layer deposition, Appl. Phys. Lett. 91, 161114 (2007).

[129] S. Kiravittaya, H. S. Lee, L. Balet, L. H. Li, M. Francardi, A. Gerardino, A. Fiore, A. Rastelli, and O. G. Schmidt, Tuning optical modes in slab photonic crystal by atomic layer deposition and laser-assisted oxidation, J. Appl. Phys. 109, 053115 (2011).

[130] Andrei Faraon, Dirk Englund, Douglas Bulla, Barry Luther-Davies, Benjamin J. Eggleton, Nick Stoltz, Pierre Petroff, and Jelena Vučković, Local tuning of photonic crystal cavities using chalcogenide glasses, Appl. Phys. Lett. 92, 043123 (2008).

[131] Srujan Meesala, Young-Ik Sohn, Benjamin Pingault, Linbo Shao, Haig A. Atikian, Jeffrey Holzgrafe, Mustafa Gündoğan, Camille Stavrakas, Alp Sipahigil, and Cleaven Chia, et al., Strain engineering of the silicon-vacancy center in diamond, Phys. Rev. B 97, 205444 (2018).

[132] Shuo Sun, Jingyuan Linda Zhang, Kevin A. Fischer, Michael J. Burek, Constantin Dory, Konstantinos G. Lagoudakis, Yan-Kai Tzeng, Marina Radulaski, Yousif Kelaita, and Amir Safavi-Naeini, et al., Cavity-Enhanced Raman Emission from a Single Color Center in a Solid, Phys. Rev. Lett. 121, 083601 (2018).

[133] C. T. Nguyen, D. D. Sukachev, M. K. Bhaskar, B. Machielse, D. S. Levonian, E. N. Knall, P. Stroganov, R. Riedinger, H. Park, and M. Lončar, et al., Quantum Network Nodes Based on Diamond Qubits with an Efficient Nanophotonic Interface, Phys. Rev. Lett. 123, 183602 (2019).

[134] Daniel Riedel, Immo Söllner, Brendan J. Shields, Sebastian Starosielec, Patrick Appel, Elke Neu, Patrick Maletinsky, and Richard J. Warburton, Deterministic Enhancement of Coherent Photon Generation from a Nitrogen-Vacancy Center in Ultrapure Diamond, Phys. Rev. X 7, 031040 (2017).

[135] David Hunger, Tilo Steinmetz, Yves Colombe, Christian Deutsch, Theodor W Hänsch, and Jakob Reichel, A fiber fabry-perot cavity with high finesse, New J. Phys. 12, 065038 (2010).

[136] Daniel Barredo, Sylvain De Léséleuc, Vincent Lienhard, Thierry Lahaye, and Antoine Browaeys, An atom-by-atom assembler of defect-free arbitrary two-dimensional atomic arrays, Science 354, 1021 (2016).

[137] Daniel Najer, Immo Söllner, Pavel Sekatski, Vincent Dolique, Matthias C Löbl, Daniel Riedel, Rüdiger Schott, Sebastian Starosielec, Sascha R. Valentin, and Andreas D. Wieck, et al., A gated quantum dot strongly coupled to an optical microcavity, Nature 575 , 1 (2019).

[138] David Hunger, Christian Deutsch, Russell J. Barbour, Richard J. Warburton, and Jakob Reichel, Laser microfabrication of concave, low-roughness features in silica, AIP Adv. 2, 012119 (2012). 
[139] Stefan Häußler, Julia Benedikter, Kerem Bray, Blake Regan, Andreas Dietrich, Jason Twamley, Igor Aharonovich, David Hunger, and Alexander Kubanek, Diamond photonics platform based on silicon vacancy centers in a single-crystal diamond membrane and a fiber cavity, Phys. Rev. B 99, 165310 (2019).

[140] Benjamin Merkel, Alexander Ulanowski, and Andreas Reiserer, Coherent emission of erbium dopants in a high-q resonator, arXiv:2006.14229 (2020).

[141] Natasha Tomm, Alisa Javadi, Nadia O. Antoniadis, Daniel Najer, Matthias C. Löbl, Alexander R. Korsch, Rüdiger Schott, Sascha R. Valentin, Andreas D. Wieck, and Arne Ludwig, et al., A bright and fast source of coherent single photons, arXiv:2007.12654 (2020).

[142] P. K. J. Singaravelu, G. C. R. Devarapu, Sebastian A. Schulz, Quentin Wilmart, Stéphane Malhouitre, and Ségolène Olivier, et al., Low-loss, compact, spot-sizeconverter based vertical couplers for photonic integrated circuits, J. Phys. D: Appl. Phys. 52, 214001 (2019).

[143] Wolfram H. P. Pernice, C. Schuck, O. Minaeva, M. Li, G. N. Goltsman, A. V. Sergienko, and H. X. Tang, Highspeed and high-efficiency travelling wave single-photon detectors embedded in nanophotonic circuits, Nat. Commun. 3, 1 (2012).

[144] Faraz Najafi, Jacob Mower, Nicholas C. Harris, Francesco Bellei, Andrew Dane, Catherine Lee, Xiaolong $\mathrm{Hu}$, Prashanta Kharel, Francesco Marsili, and Solomon Assefa, et al., On-chip detection of non-classical light by scalable integration of single-photon detectors, Nat. Commun. 6, 1 (2015).

[145] Felix Eltes, Gerardo E. Villarreal-Garcia, Daniele Caimi, Heinz Siegwart, Antonio A. Gentile, Andy Hart, Pascal Stark, Graham D. Marshall, Mark G. Thompson, and Jorge Barreto, et al., An integrated optical modulator operating at cryogenic temperatures, Nat. Mater. 19, 1 (2020).

[146] Alexander Y. Piggott, Jesse Lu, Konstantinos G. Lagoudakis, Jan Petykiewicz, Thomas M. Babinec, and Jelena Vučković, Inverse design and demonstration of a compact and broadband on-chip wavelength demultiplexer, Nat. Photonics 9, 374 (2015).

[147] Sean Molesky, Zin Lin, Alexander Y. Piggott, Weiliang Jin, Jelena Vucković, and Alejandro W. Rodriguez, Inverse design in nanophotonics, Nat. Photonics 12, 659 (2018).

[148] Logan Su, Dries Vercruysse, Jinhie Skarda, Neil V. Sapra, Jan A. Petykiewicz, and Jelena Vučković, Nanophotonic inverse design with spins: Software architecture and practical considerations, Appl. Phys. Rev. 7, 011407 (2020).

[149] Alexander Y. Piggott, Jan Petykiewicz, Logan Su, and Jelena Vučković, Fabrication-constrained nanophotonic inverse design, Sci. Rep. 7, 1 (2017).

[150] Dries Vercruysse, Neil V. Sapra, Logan Su, and Jelena Vuckovic, Dispersion engineering with photonic inverse design, IEEE J. Sel. Top. Quantum. Electron. 26, 1 (2019).

[151] Neil V. Sapra, Dries Vercruysse, Logan Su, Ki Youl Yang, Jinhie Skarda, Alexander Y. Piggott, and Jelena Vučković, Inverse design and demonstration of broadband grating couplers, IEEE J. Sel. Top. Quantum Electron. 25, 1 (2019).

[152] Constantin Dory, Dries Vercruysse, Ki Youl Yang, Neil V. Sapra, Alison E. Rugar, Shuo Sun, Daniil M. Lukin, Alexander Y. Piggott, Jingyuan L. Zhang, and Marina Radulaski, et al., Inverse-designed diamond photonics, Nat. Commun. 10, 1 (2019).

[153] Alejandro González-Tudela, C.-L. Hung, Darrick E. Chang, J. Ignacio Cirac, and H. J. Kimble, Subwavelength vacuum lattices and atom-atom interactions in two-dimensional photonic crystals, Nat. Photonics 9, 320 (2015).

[154] Alexandre Blais, Steven M. Girvin, and William D. Oliver, Quantum information processing and quantum optics with circuit quantum electrodynamics, Nat. Phys. 16, 1 (2020).

[155] Tatsuhiro Onodera, Edwin Ng, and Peter L. McMahon, A quantum annealer with fully programmable all-to-all coupling via floquet engineering, npj Quantum Inf. 6, 1 (2020). 